\title{
Axion-Higgs interplay in the two-Higgs-doublet model
}

\author{
Domènec Espriu, ${ }^{1}$ Federico Mescia, ${ }^{2}$ and Albert Renau ${ }^{1}$ \\ ${ }^{1}$ Departament d'Estructura i Constituents de la Matèria, Institut de Ciències del Cosmos (ICCUB), \\ Universitat de Barcelona, Martí Franquès 1, 08028 Barcelona, Spain \\ ${ }^{2}$ Departament de Física Fonamental, Institut de Ciències del Cosmos (ICCUB), Universitat de Barcelona, \\ Martí Franquès 1, 08028 Barcelona, Spain
}

(Received 5 August 2015; published 9 November 2015)

\begin{abstract}
The Zhitnitsky and Dine, Fischler and Srednicki (DFSZ) model is a natural extension of the two-Higgsdoublet model containing an additional singlet, endowed with a Peccei-Quinn symmetry, and leading to a physically acceptable axion. In this paper we reexamine this model in the light of some new developments. For generic couplings the model reproduces the minimal Standard Model showing only tiny deviations (extreme decoupling scenario) and all additional degrees of freedom (with the exception of the axion) are very heavy. Recently it has been remarked that the limit where the coupling between the singlet and the two doublets becomes very small is technically natural. Combining this limit with the requirement of exact or approximate custodial symmetry, we may obtain an additional $0^{+}$Higgs at the weak scale, accompanied by relatively light charged and neutral pseudoscalars. The mass spectrum would then resemble that of a generic two-Higgs-doublet model, with naturally adjustable masses in spite of the large scale that the axion introduces. However, the couplings are nongeneric in this model. We use the recent constraints derived from the Higgs- $W W$ coupling together with oblique corrections to constrain the model as much as possible. As an additional result, we work out the nonlinear parametrization of the DFSZ model in the generic case where all scalars except the lightest Higgs and the axion have masses at or beyond the TeV scale.
\end{abstract}

DOI: 10.1103/PhysRevD.92.095013

PACS numbers: $12.60 . \mathrm{Fr}, 14.80 . \mathrm{Bn}, 14.80 . \mathrm{Ec}, 95.35 .+\mathrm{d}$

\section{INTRODUCTION}

An invisible axion [1-3] constitutes to this date a very firm candidate to provide all or part of the dark matter component of the cosmological budget. There are several extensions of the minimal Standard Model (MSM) providing a particle with the characteristics and couplings of the axion $[4,5]$. In our view a particularly interesting possibility is the model suggested by Zhitnitsky and Dine, Fischler and Srednicki (DFSZ) more than 30 years ago that consists of a fairly simple extension of the popular two-Higgs-doublet model (2HDM). As a matter of fact, it could probably be argued that a good motivation to consider the $2 \mathrm{HDM}$ is that it allows for the inclusion of a (nearly) invisible axion [6-8]. Of course, there are other reasons why the 2HDM should be considered as a possible extension of the MSM. Apart from purely aesthetic reasons, it is easy to reconcile such models with existing constraints. They may give rise to a rich phenomenology, including possibly (but not necessarily) flavor-changing neutral currents at some level, custodial symmetry breaking terms or even new sources of $C P$ violation $[9,10]$.

Following the discovery of a Higgs-like particle with $m_{h} \sim 125 \mathrm{GeV}$, there have been a number of works considering the implications of such a finding on a generic $2 \mathrm{HDM}$, together with the constraints arising from the lack of detection of new scalars and from the electroweak precision observables [11]. Depending on the way that the two doublets couple to fermions, they are classified as type I, II or III (see e.g. [9] for details), with different implications on the flavor sector. Consideration of all the different types of $2 \mathrm{HDM}$ plus all the rich phenomenology that can be encoded in the Higgs potential leads to a wide variety of possibilities with different experimental implications, even after applying all the known phenomenological low-energy requirements.

Requiring a Peccei-Quinn (PQ) symmetry leading to an axion does, however, severely restrict the possibilities, and this is in our view an important asset of the DFSZ model. This turns out to be particularly the case when one includes all the recent experimental information concerning the $125 \mathrm{GeV}$ scalar state and its couplings. Exploring this model, taking into account all these constraints is the motivation for the present work.

The structure of this paper is as follows. In Sec. II we discuss the possible global symmetries of the DFSZ model, namely $U(1)_{\mathrm{PQ}}$ (always present), and $S U(2)_{L} \times S U(2)_{R}$ (the $S U(2)_{R}$ subgroup may or may not be present). Symmetries are best discussed by using a matrix formalism that we review and extend. Section III is devoted to the determination of the spectrum of the theory. We present up to four generic cases that range from the extreme decoupling, where the model-apart from the presence of the axion-is indistinguishable from the MSM at low energies, to one where there are extra light Higgses below or around the $\mathrm{TeV}$ scale. This last case necessarily requires some couplings in the potential to be very small; a possibility that is nevertheless natural in a technical sense and therefore 
should be contemplated as a viable theoretical hypothesis. We discuss in detail the situation where custodial symmetry is exact or approximately valid in this model because the combination of this symmetry and naturally small couplings allows us to keep the additional scalars 'naturally light' if we so wish with only one exception, meaning that the 'contamination' from the large scale present in the theory is under control.

However, while additional scalars may exist at or just above the weak scale in this model, they can also be made heavy, with masses in the multi- $\mathrm{TeV}$ region or beyond. In Sec. IV we discuss the resulting nonlinear effective theory emerging in this generic situation.

Next in Sec. V we analyze the impact of the model on the (light) Higgs effective couplings to gauge bosons and the constraints that can be derived from the recent LHC data. In Sec. VI we compare the potential of the DFSZ model with the most general potential in the 2HDM. We find out which terms of the general potential are forbidden by the PQ symmetry and which ones are recovered when it is spontaneously broken by the VEV of the scalar fields. Finally in Sec. VII the restrictions that the electroweak precision parameters, particularly $\Delta \rho$, impose on the model are discussed. These restrictions are relevant only in the case where all or part of the additional spectrum of scalars is light as we find that they are automatically satisfied otherwise.

We would like to emphasize that even after imposing the constraints derived from the PQ symmetry the model still contains enough degrees of freedom to reproduce the mass spectrum of a generic $2 \mathrm{HDM}$, so there is no predictivity at the level of the spectrum. However, this nearly exhausts all freedom available, particularly if exact or approximate custodial symmetry is imposed. Then the scalar couplings are largely fixed and in this sense the model is far more predictive than a generic $2 \mathrm{HDM}$ - and in addition it contains the axion, which is its raison d'être.

\section{MODEL AND SYMMETRIES}

The DFSZ model contains two Higgs doublets and one complex scalar singlet, namely,

$$
\phi_{1}=\left(\begin{array}{c}
\alpha_{+} \\
\alpha_{0}
\end{array}\right) ; \quad \phi_{2}=\left(\begin{array}{c}
\beta_{+} \\
\beta_{0}
\end{array}\right) ; \quad \phi,
$$

with vacuum expectation values (VEVs) $\left\langle\alpha_{0}\right\rangle=v_{1}$, $\left\langle\beta_{0}\right\rangle=v_{2},\langle\phi\rangle=v_{\phi}$ and $\left\langle\alpha_{+}\right\rangle=\left\langle\beta_{+}\right\rangle=0$. Moreover, we define the usual electroweak vacuum expectation value $v=246 \mathrm{GeV}$ as $v^{2}=\left(v_{1}^{2}+v_{2}^{2}\right) / 2$ and $\tan \beta=v_{2} / v_{1}$. The implementation of the PQ symmetry is only possible for type II models, where the Yukawa terms are

$$
\mathcal{L}_{Y}=G_{1} \bar{q}_{L} \tilde{\phi}_{1} u_{R}+G_{2} \bar{q}_{L} \phi_{2} d_{R}+\text { H.c. },
$$

with $\tilde{\phi}_{i}=i \tau_{2} \phi_{i}^{*}$. The PQ transformation acts on the scalars as $\phi_{1} \rightarrow e^{i X_{1} \theta} \phi_{1}, \quad \phi_{2} \rightarrow e^{i X_{2} \theta} \phi_{2}, \quad \phi \rightarrow e^{i X_{\phi} \theta} \phi$

and on the fermions as

$q_{L} \rightarrow q_{L}, \quad l_{L} \rightarrow l_{L}, \quad u_{R} \rightarrow e^{i X_{u} \theta} u_{R}$,

$d_{R} \rightarrow e^{i X_{d} \theta} d_{R}, \quad e_{R} \rightarrow e^{i X_{e} \theta} e_{R}$.

For the Yukawa terms to be PQ-invariant we need

$$
X_{u}=X_{1}, \quad X_{d}=-X_{2}, \quad X_{e}=-X_{2} .
$$

Let us now turn to the potential involving the two doublets and the new complex singlet. The most general potential compatible with PQ symmetry is

$$
\begin{aligned}
V\left(\phi, \phi_{1}, \phi_{2}\right)= & \lambda_{\phi}\left(\phi^{*} \phi-V_{\phi}^{2}\right)^{2}+\lambda_{1}\left(\phi_{1}^{\dagger} \phi_{1}-V_{1}^{2}\right)^{2} \\
& +\lambda_{2}\left(\phi_{2}^{\dagger} \phi_{2}-V_{2}^{2}\right)^{2} \\
& +\lambda_{3}\left(\phi_{1}^{\dagger} \phi_{1}-V_{1}^{2}+\phi_{2}^{\dagger} \phi_{2}-V_{2}^{2}\right)^{2} \\
& +\lambda_{4}\left[\left(\phi_{1}^{\dagger} \phi_{1}\right)\left(\phi_{2}^{\dagger} \phi_{2}\right)-\left(\phi_{1}^{\dagger} \phi_{2}\right)\left(\phi_{2}^{\dagger} \phi_{1}\right)\right] \\
& +\left(a \phi_{1}^{\dagger} \phi_{1}+b \phi_{2}^{\dagger} \phi_{2}\right) \phi^{*} \phi \\
& -c\left(\phi_{1}^{\dagger} \phi_{2} \phi^{2}+\phi_{2}^{\dagger} \phi_{1} \phi^{* 2}\right)
\end{aligned}
$$

The $c$ term imposes the condition $-X_{1}+X_{2}+2 X_{\phi}=0$. If we impose that the PQ current does not couple to the Goldstone boson that is eaten by the $Z$, we also get $X_{1} \cos ^{2} \beta+X_{2} \sin ^{2} \beta=0$. If furthermore we choose ${ }^{1}$ $X_{\phi}=-1 / 2$ the PQ charges of the doublets are

$$
X_{1}=-\sin ^{2} \beta, \quad X_{2}=\cos ^{2} \beta .
$$

Global symmetries are not very evident in the way fields are introduced above. To remedy this let us define the matrices [12]

$$
\begin{aligned}
& \Phi_{12}=\left(\tilde{\phi}_{1} \phi_{2}\right)=\left(\begin{array}{cc}
\alpha_{0}^{*} & \beta_{+} \\
-\alpha_{-} & \beta_{0}
\end{array}\right), \\
& \Phi_{21}=\left(\tilde{\phi}_{2} \phi_{1}\right)=\left(\begin{array}{cc}
\beta_{0}^{*} & \alpha_{+} \\
-\beta_{-} & \alpha_{0}
\end{array}\right)=\tau_{2} \Phi_{12}^{*} \tau_{2}
\end{aligned}
$$

and

$$
\begin{aligned}
& I=\Phi_{12}^{\dagger} \Phi_{12}=\left(\begin{array}{cc}
\phi_{1}^{\dagger} \phi_{1} & \tilde{\phi}_{1}^{\dagger} \phi_{2} \\
-\phi_{1}^{\dagger} \tilde{\phi}_{2} & \phi_{2}^{\dagger} \phi_{2}
\end{array}\right), \\
& J=\Phi_{12}^{\dagger} \Phi_{21}=\phi_{2}^{\dagger} \phi_{1} \mathbf{I} .
\end{aligned}
$$

Defining also the constant matrix $W=\left(V_{1}^{2}+V_{2}^{2}\right) \mathbf{I} / 2+$ $\left(V_{1}^{2}-V_{2}^{2}\right) \tau_{3} / 2$, we can write the potential as

\footnotetext{
${ }^{1}$ There is arbitrariness in this choice. This election conforms to the conventions existing in the literature.
} 


$$
\begin{aligned}
V(\phi, I, J)= & \lambda_{\phi}\left(\phi^{*} \phi-V_{\phi}^{2}\right)^{2}+\frac{\lambda_{1}}{4}\left\{\operatorname{Tr}\left[(I-W)\left(1+\tau_{3}\right)\right]\right\}^{2} \\
& +\frac{\lambda_{2}}{4}\left\{\operatorname{Tr}\left[(I-W)\left(1-\tau_{3}\right)\right]\right\}^{2}+\lambda_{3}[\operatorname{Tr}(I-W)]^{2} \\
& +\frac{\lambda_{4}}{4} \operatorname{Tr}\left[I^{2}-\left(I \tau_{3}\right)^{2}\right] \\
& +\frac{1}{2} \operatorname{Tr}\left[(a+b) I+(a-b) I \tau_{3}\right] \phi^{*} \phi \\
& -\frac{c}{2} \operatorname{Tr}\left(J \phi^{2}+J^{\dagger} \phi^{* 2}\right) .
\end{aligned}
$$

A $S U(2)_{L} \times S U(2)_{R}$ global transformation acts on our fields as

$$
\Phi_{i j} \rightarrow L \Phi_{i j} R^{\dagger}, \quad I \rightarrow R I R^{\dagger}, \quad J \rightarrow J .
$$

We now we are in a better position to discuss the global symmetries of the potential. The behavior of the different parameters under $S U(2)_{R}$ is shown in Table I. See also [13].

Finally, let us establish the action of the PQ symmetry previously discussed in this parametrization. Under the PQ transformation:

$$
\Phi_{12} \rightarrow \Phi_{12} e^{i X \theta}, \quad \phi \rightarrow e^{i X_{\phi} \theta} \phi
$$

with

$X=\frac{X_{2}-X_{1}}{2} \mathbf{I}-\frac{X_{2}+X_{1}}{2} \tau_{3}, \quad X_{\phi}=\frac{X_{2}-X_{1}}{2}$

Using the values for $X_{1,2}$ given in Eq. (7)

$$
X=\left(\begin{array}{cc}
\sin ^{2} \beta & 0 \\
0 & \cos ^{2} \beta
\end{array}\right), \quad X_{\phi}=-\frac{1}{2} .
$$

\section{MASSES AND MIXINGS}

We have two doublets and a singlet, so a total of $4+4+2=10$ spin-zero particles. Three particles are eaten by the $W^{ \pm}$and $Z$ and 7 scalars fields are left in the spectrum; two charged Higgs, two $0^{-}$states and three neutral $0^{+}$states. Our field definitions will be worked out in full detail in Sec. IV. Here we want only to derive the spectrum. For the charged Higgs mass we have at tree level, ${ }^{2}$

$$
m_{H_{ \pm}}^{2}=8\left(\lambda_{4} v^{2}+\frac{c v_{\phi}^{2}}{s_{2 \beta}}\right) .
$$

The quantity $v_{\phi}$ is proportional to the axion decay constant. Its value is known to be very large (at least

\footnotetext{
${ }^{2}$ Here and in the following we introduce the short-hand notation $s_{m \beta}^{n} \equiv \sin ^{n}(m \beta)$ and $c_{m \beta}^{n} \equiv \cos ^{n}(m \beta)$.
}

$10^{7} \mathrm{GeV}$ and probably substantially larger $\sim 10^{9} \mathrm{GeV}$ if all astrophysical constraints are taken into account, see [14] for several experimental and cosmological bounds). It does definitely make sense to organize the calculations as an expansion in $v / v_{\phi}$.

In the $0^{-}$sector there are two degrees of freedom that mix with each other with a mass matrix which has a vanishing eigenvalue. The eigenstate with zero mass is the axion and $A_{0}$ is the pseudoscalar Higgs with mass

$$
m_{A_{0}}^{2}=8 c\left(\frac{v_{\phi}^{2}}{s_{2 \beta}}+v^{2} s_{2 \beta}\right)
$$

Eq. (16) implies $c \geq 0$. For $c=0$, the mass matrix in the $0^{-}$ sector has a second zero, i.e. in practice the $A_{0}$ field behaves as another axion.

In the $0^{+}$sector, there are three neutral particles that mix with each other. With $h_{i}$ we denote the corresponding $0^{+}$ mass eigenstates. The mass matrix is given in Appendix B. In the limit of large $v_{\phi}$, the mass matrix in the $0^{+}$sector can be easily diagonalized [7] and presents one eigenvalue nominally of order $v^{2}$ and two of order $v_{\phi}^{2}$. Up to $\mathcal{O}\left(v^{2} / v_{\phi}^{2}\right)$, these masses are

$$
\begin{aligned}
& m_{h_{1}}^{2}= 32 v^{2}\left(\lambda_{1} c_{\beta}^{4}+\lambda_{2} s_{\beta}^{4}+\lambda_{3}\right) \\
&-16 v^{2} \frac{\left(a c_{\beta}^{2}+b s_{\beta}^{2}-c s_{2 \beta}\right)^{2}}{\lambda_{\phi}}, \\
& m_{h_{2}}^{2}= \frac{8 c}{s_{2 \beta}} v_{\phi}^{2}+8 v^{2} s_{2 \beta}^{2}\left(\lambda_{1}+\lambda_{2}\right) \\
&-4 v^{2} \frac{\left[(a-b) s_{2 \beta}+2 c c_{2 \beta}\right]^{2}}{\lambda_{\phi}-2 c / s_{2 \beta}}, \\
& m_{h_{3}}^{2}=4 \lambda_{\phi} v_{\phi}^{2}+4 v^{2} \frac{\left[(a-b) s_{2 \beta}+2 c c_{2 \beta}\right]^{2}}{\lambda_{\phi}-2 c / s_{2 \beta}} \\
&+16 v^{2} \frac{\left(a c_{\beta}^{2}+b s_{\beta}^{2}-c s_{2 \beta}\right)^{2}}{\lambda_{\phi}} .
\end{aligned}
$$

The field $h_{1}$ is naturally identified with the scalar boson of mass $125 \mathrm{GeV}$ observed at the LHC.

It is worth it to stress that there are several situations where the above formulae are nonapplicable, since the nominal expansion in powers of $v / v_{\phi}$ may fail. This may be the case where the coupling constants $a, b, c$ connecting the singlet to the usual 2HDM are very small, of order say $v / v_{\phi}$ or $v^{2} / v_{\phi}^{2}$. One should also pay attention to the case $\lambda_{\phi} \rightarrow 0$ (we have termed this latter case as the "quasifree singlet limit"). Leaving this last case aside, we have found that the above expressions for $m_{h_{i}}$ apply in the following situations:

Case 1: The couplings $a, b$ and $c$ are generically of $\mathcal{O}(1)$ 
Case 2: $a, b$ or $c$ are of $\mathcal{O}\left(v / v_{\phi}\right)$

Case 3: $a, b$ or $c$ are of $\mathcal{O}\left(v^{2} / v_{\phi}^{2}\right)$ but $c \gg \lambda_{i} v^{2} / v_{\phi}^{2}$.

If $c \ll \lambda_{i} v^{2} / v_{\phi}^{2}$ the $0^{-}$state is lighter than the lightest $0^{+}$ Higgs and this case is therefore already phenomenologically unacceptable. The only other case that deserves a separate discussion is

Case 4: Same as in case 3 but $c \sim \lambda_{i} v^{2} / v_{\phi}^{2}$.

In this case, the masses in the $0^{+}$sector read, up to $\mathcal{O}\left(v^{2} / v_{\phi}^{2}\right)$, as

$m_{h_{1}, h_{2}}^{2}=8 v^{2}\left(K \mp \sqrt{K^{2}-L}\right) \quad$ and $\quad m_{h_{3}}^{2}=4 \lambda_{\phi} v_{\phi}^{2}$,

where

$$
\begin{aligned}
K & =2\left(\lambda_{1} c_{\beta}^{2}+\lambda_{2} s_{\beta}^{2}+\lambda_{3}\right)+\frac{c v_{\phi}^{2}}{2 v^{2} s_{2 \beta}}, \\
L & =4\left[\left(\lambda_{1} \lambda_{2}+\lambda_{1} \lambda_{3}+\lambda_{2} \lambda_{3}\right) s_{2 \beta}^{2}+\frac{c v_{\phi}^{2}}{v^{2} s_{2 \beta}}\left(\lambda_{1} c_{\beta}^{4}+\lambda_{2} s_{\beta}^{4}+\lambda_{3}\right)\right] .
\end{aligned}
$$

Recall that here we assume $c$ to be of $\mathcal{O}\left(v^{2} / v_{\phi}^{2}\right)$. Note that

$m_{h_{1}}^{2}+m_{h_{2}}^{2}=32 v^{2}\left(\lambda_{1} c_{\beta}^{2}+\lambda_{2} s_{\beta}^{2}+\lambda_{3}+\frac{c v_{\phi}^{2}}{4 v^{2} s_{2 \beta}}\right)$.

In the "quasifree singlet" limit, when $\lambda_{\phi} \rightarrow 0$ or more generically $\lambda_{\phi} \ll a, b, c$ it is impossible to sustain the hierarchy $v \ll v_{\phi}$, so again this case is phenomenologically uninteresting (see Appendix $\mathrm{C}$ for details).

We note that once we set $\tan \beta$ to a fixed value, the lightest Higgs to $125 \mathrm{GeV}$ and $v_{\phi}$ to some large value compatible with the experimental bounds, the mass spectrum in Eq. (15), (16) and (17)-(19) grossly depends on the parameters: $c, \lambda_{4}$ and $\lambda_{\phi}$, the latter only affecting the third $0^{+}$state that is anyway very heavy and definitely out of reach of LHC experiments; therefore the spectrum depends on only two parameters. If case 4 is applicable, the situation is slightly different and an additional combination of parameters dictates the mass of the second (lightish) $0^{+}$ state. This can be seen in the sum rule of Eq. (22) after requiring that $m_{h_{1}}=125 \mathrm{GeV}$. Actually this sum rule is also obeyed in cases 1,2 and 3, but the r.h.s is dominated then by the contribution from the parameter $c$ alone.

\section{A. Heavy and light states}

Here we want to discuss the spectrum of the theory according to the different scenarios that we have alluded to in the previous discussion. Let us remember that it is always possible to identify one of the Higgses as the scalar boson found at LHC, namely $h_{1}$.

Case 1. all Higgses except $h_{1}$ acquire a mass of order $v_{\phi}$. This includes the charged and $0^{-}$scalars, too. We term this situation 'extreme decoupling'. The only light
TABLE I. In total, there are 11 parameters: 7 are custodially preserving and 4 are custodially breaking. See the text for our usage of the expression 'custodial symmetry' in the context of a 2HDM.

\begin{tabular}{lc}
\hline \hline Parameter & Custodial limit \\
\hline$\lambda_{1}, \lambda_{2}, \lambda_{4}$ & $\lambda_{1}=\lambda_{2}=\lambda$ and $\lambda_{4}=2 \lambda$ \\
$\lambda_{3}$ & $\lambda_{3}$ \\
$\lambda_{\phi}$ & $\lambda_{\phi}$ \\
$V_{1}^{2}, V_{2}^{2}$ & $V_{1}^{2}=V_{2}^{2}=V^{2}$ \\
$V_{\phi}$ & $V_{\phi}$ \\
$a, b$ & $a=b$ \\
$c$ & $c$ \\
\hline \hline
\end{tabular}

states are $h_{1}$, the gauge sector and the massless axion. This is the original DFSZ scenario [4]

Case 2. This situation is similar to case 1 but now the typical scale of masses of $h_{2}, H_{ \pm}$and $A_{0}$ is $\sqrt{v v_{\phi}}$. This range is beyond the LHC reach but it could perhaps be explored with an accelerator in the $100 \mathrm{TeV}$ region, a possibility being currently debated. Again the only light particles are $h_{1}$, the axion and the gauge sector. This possibility is natural in a technical sense as discussed in [7] as an approximate extra symmetry would protect the hierarchy.

Cases 3 and 4 are phenomenologically more interesting. Here we can at last have new states at the weak scale. In the $0^{+}$sector, $h_{3}$ is definitely very heavy but $m_{h_{1}}^{2}$ and $m_{h_{2}}^{2}$ are proportional to $v^{2}$ once we assume that $c \sim v^{2} / v_{\phi}^{2}$. Depending on the relative size of $\lambda_{i}$ and $c v_{\phi}^{2} / v^{2}$ one would have to use Eq. (17) or (20). Because in case 3 one assumes that $c v_{\phi}^{2} / v^{2}$ is much larger than $\lambda_{i}, h_{1}$ would still be the lightest Higgs and $m_{h_{2}}$ could easily be in the TeV region. When examining case 4 it would be convenient to use the sum rule (22).

We note that in case 4 the hierarchy between the different couplings is quite marked: typically to be realized one needs $c \sim 10^{-10} \lambda_{i}$, where $\lambda_{i}$ is a generic coupling of the potential. It is the smallness of this number what results in the presence of light states at the weak scale. For a discussion on the "naturalness" of this possibility see [7].

\section{B. Custodially symmetric potential}

While in the usual one doublet model, if we neglect the Yukawa couplings and set the $U_{Y}(1)$ interactions to zero, custodial symmetry is automatic, the latter is somewhat unnatural in 2HDM as one can write a fairly general potential. These terms are generically not invariant under global transformations $S U(2)_{L} \times S U(2)_{R}$ and therefore in the general case after the breaking there is no custodial symmetry to speak of. Let us consider now the case where a global symmetry $S U(2)_{L} \times S U(2)_{R}$ is nevertheless present as there are probably good reasons to consider this limit. 
We may refer somewhat improperly to this situation as to being 'custodially symmetric' although after the breaking custodial symmetry proper may or may not be present. If $S U(2)_{L} \times S U(2)_{R}$ is to be a symmetry, the parameters of the potential have to be set according to the custodial relations in Table I. Now, there are two possibilities to spontaneously break $S U(2)_{L} \times S U(2)_{R}$ and to give mass to the gauge bosons.$$
\text { 1. } S U(2) \times S U(2) \rightarrow U(1)
$$

If the VEVs of the two Higgs fields are different $(\tan \beta \neq 1)$, the custodial symmetry is spontaneously broken to $U(1)$. In this case, one can use the minimization equations of Appendix A to eliminate $V, V_{\phi}$ and $c$ of Eq. (10). $c$ turns out to be of order $\left(v / v_{\phi}\right)^{2}$. In this case there are two extra Goldstone bosons: the charged Higgs is massless

$$
m_{H_{ \pm}}^{2}=0 .
$$

Furthermore, the $A_{0}$ is light:

$$
m_{A_{0}}^{2}=16 \lambda v^{2}\left(1+\frac{v^{2}}{v_{\phi}^{2}} s_{2 \beta}^{2}\right)
$$

This situation is clearly phenomenologically excluded.

$$
\text { 2. } S U(2)_{L} \times S U(2)_{R} \rightarrow S U(2)_{V}
$$

In this case, the VEVs of the Higgs doublets are equal, so $\tan \beta=1$. The masses are

$$
m_{H_{ \pm}}^{2}=8\left(2 \lambda v^{2}+c v_{\phi}^{2}\right), \quad m_{A_{0}}^{2}=8 c\left(v^{2}+v_{\phi}^{2}\right)
$$

and $m_{h_{2}}^{2}=m_{H_{ \pm}}^{2}$.

These three states are parametrically heavy, but they may be light in cases 3 and 4 . The rest of the $0^{+}$mass matrix is $2 \times 2$ and has eigenvalues (up to second order in $v / v_{\phi}$ )

$$
\begin{aligned}
& m_{h_{1}}^{2}=16 v^{2}\left[\lambda+2 \lambda_{3}-\frac{(a-c)^{2}}{\lambda_{\phi}}\right] \text { and } \\
& m_{h_{3}}^{2}=4\left[\lambda_{\phi} v_{\phi}^{2}+4 v^{2} \frac{(a-c)^{2}}{\lambda_{\phi}}\right] .
\end{aligned}
$$

It is interesting to explore in this relatively simple case what sort of masses can be obtained by varying the values of the couplings in the potential $\left(\lambda, \lambda_{3}\right.$ and $\left.c\right)$. We are basically interested in the possibility of obtaining a lightish spectrum (case 4 previously discussed) and accordingly we assume that the natural scale of $c$ is $\sim v^{2} / v_{\phi}^{2}$. We have to require the stability of the potential discussed in Appendix D as well as $m_{h_{1}}=125 \mathrm{GeV}$. The allowed region is shown in Fig. 1. Since we are in a custodially symmetric case there are no further restrictions to be obtained from $\Delta \rho$.

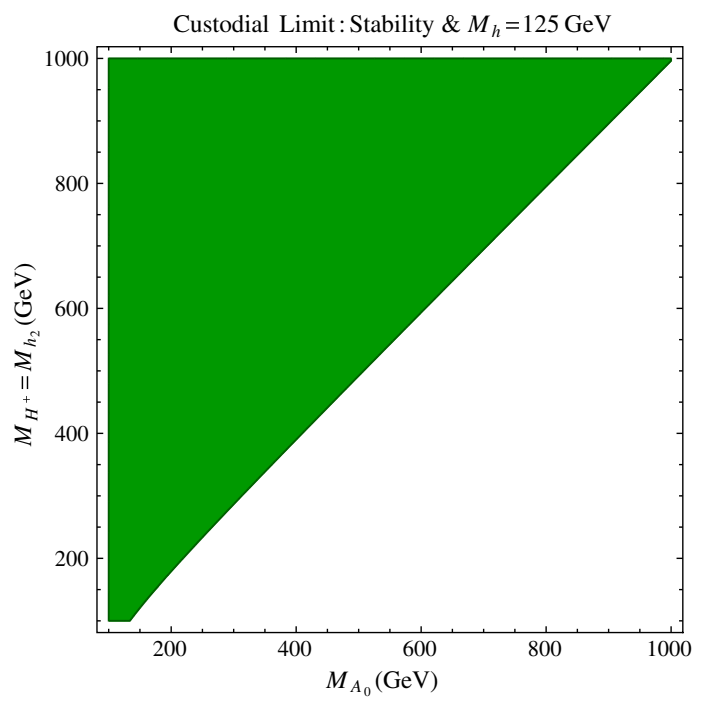

FIG. 1 (color online). Dark/green: allowed region in the custodial limit after requiring vacuum stability (see e.g. Appendix D). Each point in this region corresponds to a valid set of parameters in the DFSZ potential. Note that $c$ is assumed to be of order $v^{2} / v_{\phi}^{2}$ and $c v_{\phi}^{2} / v^{2}$ has to be $\sim \lambda_{i}$ (case 4 discussed in the text).

\section{Understanding hierarchies}

As is well known in the MSM the Higgs mass has potentially large corrections if the MSM is understood as an effective theory and one assumes that a larger scale must show up in the theory at some moment. This is the case, for instance, if neutrino masses are included via the see-saw mechanism, to name just a possibility. In this case to keep the $125 \mathrm{GeV}$ Higgs light one must do some amount of fine-tuning.

In the DFSZ model such a large scale is indeed present from the outset and consequently one has to envisage the possibility that the mass formulae previously derived may be subject to large corrections due to the fact that $v_{\phi}$ leaks in the low-energy scalar spectrum. Let us discuss the relevance of the hierarchy problem in the different cases discussed in this section.

In case 1 all masses in the scalar sector but the physical Higgs are heavy, of order $v_{\phi}$, and due to the fact that the couplings $\lambda_{i}$ in the potential are generic (and also the couplings $a, b, c$ connecting the two doublets to the singlet) the hierarchy may affect the light Higgs quite severely and fine-tuning of the $\lambda_{i}$ will be called for. However, this finetuning is not essentially different from the one commonly accepted to be necessary in the MSM to keep the Higgs light if a new scale is somehow present.

In cases 3 and 4 the amount of additional fine-tuning needed is none or very little. In these scenarios (particularly in case 4) the scalar spectrum is light, in the $\mathrm{TeV}$ region, and the only heavy degree of freedom is contained in the modulus of the singlet. After diagonalization this results in a very heavy $0^{+}$state $\left(h_{3}\right)$, with a mass or order $v_{\phi}$. 
However, inspection of the potential reveals that this degree of freedom couples to the physical sector with an strength $v^{2} / v_{\phi}^{2}$ and therefore may change the tree-level masses by a contribution of order $v$-perfectly acceptable. In this sense the "natural" scenario proposed in [7] does not apparently lead to a severe hierarchy problem in spite of the large scale involved.

Case 2 is particularly interesting. In this case the intermediate masses are of order $\sqrt{v v_{\phi}}$, i.e. $\sim 100 \mathrm{TeV}$. There is still a very heavy mass eigenstate $\left(h_{3}\right)$ but again is nearly decoupled from the lightest Higgs as in cases 3 and 4. On the contrary, the states with masses $\sim \sqrt{v v_{\phi}}$ do couple to the light Higgs with strength $\sim \lambda_{i}$ and thus requirethanks to the loop suppression factor-only a very moderate amount of fine-tuning as compared to case 1 .

It is specially relevant in the context of the hierarchy problem to consider the custodial case discussed in the previous section. In the custodial limit, the $A_{0}$ mass is protected as it is proportional to the extended symmetry breaking parameter $c$. In addition $m_{h_{2}}=m_{H^{ \pm}}$. Should one wish to keep a control on radiative corrections, doing the fine-tuning necessary to keep $h_{1}$ and $h_{2}$ light should suffice and in fact the contamination from the heavy $h_{3}$ is limited as said above. Of course, to satisfy the present data we have to worry only about $h_{1}$.

\section{NONLINEAR EFFECTIVE LAGRANGIAN}

We have seen in the previous section that the spectrum of scalars resulting from the potential of the DFSZ model is generically heavy. It is somewhat difficult to have all the scalar masses at the weak scale, although the additional scalars can be made to have masses in weak scale region in case 4 . The only exceptions are the three Goldstone bosons, the $h_{1}$ Higgs and the axion. It is therefore somehow natural to use a nonlinear realization to describe the low-energy sector formed by gauge bosons (and their associated Goldstone bosons), the lightest Higgs $0^{+}$state $h_{1}$, and the axion. Deriving this effective action is one of the motivations of this work.

To construct the effective action we have to identify the proper variables and in order to do so we will follow the technique described in [12]. In that paper the case of a generic 2HDM where all scalar fields were very massive was considered. Now we have to modify the method to allow for a light state (the $h_{1}$ ) and also to include the axion degree of freedom.

We decompose the matrix-valued $\Phi_{12}$ field introduced in Sec. II in the following form:

$$
\Phi_{12}=\mathcal{U} \mathcal{M}_{12}
$$

$\mathcal{U}$ is a $2 \times 2$ matrix that contains the three Goldstone bosons associated to the breaking of $S U(2)_{L}$ (or more precisely of $S U(2)_{L} \times U(1)_{Y}$ to $\left.U(1)_{e m}\right)$. We denote by $G^{i}$ these Goldstone bosons:

$$
\mathcal{U}=\exp \left(i \frac{\vec{G} \cdot \vec{\tau}}{v}\right)
$$

Note that the matrices $I$ and $J$ of Eq. (9) entering the DFSZ potential are actually independent of $\mathcal{U}$. This is immediate to see in the case of $I$ while for $J$ one has to use the property $\tau_{2} \mathcal{U}^{*}=\mathcal{U} \tau_{2}$ valid for $S U(2)$ matrices. The effective potential then does depend only on the degrees of freedom contained in $\mathcal{M}_{12}$ whereas the Goldstone bosons drop from the potential, since, under a global $S U(2)_{L} \times S U(2)_{R}$ rotation, $\Phi_{12}$ and $\mathcal{U}$ transform as

$\Phi_{12} \rightarrow L \Phi_{12} R^{\dagger} \quad \mathcal{U} \rightarrow L \mathcal{U} R^{\dagger} \Rightarrow \mathcal{M}_{12} \rightarrow R \mathcal{M}_{12} R^{\dagger}$.

Obviously, the same applies to the locally gauged subgroup.

Let us now discuss the potential and $\mathcal{M}_{12}$ further. Inspection of the potential shows that because of the term proportional to $c$ the phase of the singlet field $\phi$ does not drop automatically from the potential and thus it cannot be immediately identified with the axion. In other words, the phase of the $\phi$ field mixes with the usual $0^{-}$scalar from the 2HDM. To deal with this let us find a suitable phase both in $\mathcal{M}_{12}$ and in $\phi$ that drops from the effective potential-this will single out the massless state to be identified with the axion.

We write $\mathcal{M}_{12}=M_{12} U_{a}$, where $U_{a}$ is a unitary matrix containing the axion. An immediate choice is to take the generator of $U_{a}$ to be the identity, which obviously can remove the phase of the singlet in the term in the effective potential proportional to $c$ while leaving the other terms manifestly invariant. This does not exhaust all freedom, however, as we can include in the exponent of $U_{a}$ a term proportional to $\tau_{3}$. It can be seen immediately that this would again drop from all the terms in the effective potential, including the one proportional to $c$ when taking into account that $\phi$ is a singlet under the action of $\tau_{3}$ that of course is nothing but the hypercharge generator. We will use the remaining freedom just discussed to properly normalize the axion and $A_{0}$ fields in the kinetic terms to which we now turn.

The gauge invariant kinetic term will be

$$
\mathcal{L}_{\text {kin }}=\frac{1}{2}\left(\partial_{\mu} \phi\right)^{*} \partial^{\mu} \phi+\frac{1}{4} \operatorname{Tr}\left[\left(D_{\mu} \Phi_{12}^{\dagger}\right) D^{\mu} \Phi_{12}\right],
$$

where the covariant derivative is defined by

$$
D_{\mu} \Phi_{12}=\partial_{\mu} \Phi_{12}-i \frac{g}{2} \vec{W}_{\mu} \cdot \vec{\tau} \Phi_{12}+i \frac{g^{\prime}}{2} B_{\mu} \Phi_{12} \tau_{3} .
$$

By defining $U_{a}=\exp \left(2 i a_{\phi} X / \sqrt{v_{\phi}^{2}+v^{2} s_{2 \beta}^{2}}\right)$ with $X$ in Eq. (14), all terms in the kinetic term are diagonal and 
exhibit the canonical normalization. Moreover the field $a_{\phi}$ disappears from the potential. Note that the phase redefinition implied in $U_{a}$ exactly coincides with the realization of the PQ symmetry on $\Phi_{12}$ in Eq. (12) as is to be expected (this identifies uniquely the axion degree of freedom).
Finally, the nonlinear parametrization of $\Phi_{12}$ reads as

$$
\Phi_{12}=\mathcal{U} M_{12} U_{a}
$$

with

$$
M_{12}=\sqrt{2}\left(\begin{array}{cc}
(v+H) c_{\beta}-\left(S-i \frac{v_{\phi}}{\sqrt{v_{\phi}^{2}+v^{2} s_{2 \beta}^{2}}} A_{0}\right) s_{\beta} & \sqrt{2} H_{+} c_{\beta} \\
\sqrt{2} H_{-} s_{\beta} & (v+H) s_{\beta}+\left(S+i \frac{v_{\phi}}{\sqrt{v_{\phi}^{2}+v^{2} s_{2 \beta}^{2}}} A_{0}\right) c_{\beta}
\end{array}\right)
$$

and

$$
\begin{aligned}
v+H & =\frac{c_{\beta}}{\sqrt{2}} \Re\left[\alpha_{0}\right]+\frac{s_{\beta}}{\sqrt{2}} \Re\left[\beta_{0}\right], \\
S & =-\frac{s_{\beta}}{\sqrt{2}} \Re\left[\alpha_{0}\right]+\frac{c_{\beta}}{\sqrt{2}} \Re\left[\beta_{0}\right], \\
H_{ \pm} & =\frac{c_{\beta} \beta_{ \pm}-s_{\beta} \alpha_{ \pm}}{2}
\end{aligned}
$$

in terms of the fields in Eq. (1). The singlet field $\phi$ is nonlinearly parametrized as

$\phi=\left(v_{\phi}+\rho-i \frac{v s_{2 \beta}}{\sqrt{v_{\phi}^{2}+v^{2} s_{2 \beta}^{2}}} A_{0}\right) \exp \left(i \frac{a_{\phi}}{\sqrt{v_{\phi}^{2}+v^{2} s_{2 \beta}^{2}}}\right)$.

With the parametrizations above the kinetic term is diagonal in terms of the fields of $M_{12}$ and $\rho$. Moreover, the potential is independent of the axion and Goldstone bosons. All the fields appearing in Eqs. (33) and (35) have vanishing VEVs.

Let us stress that $H, S$ and $\rho$ are not mass eigenstates and their relations with the $h_{i}$ mass eigenstates are defined through

$H=\sum_{i=1}^{3} R_{H i} h_{i}, \quad S=\sum_{i=1}^{3} R_{S i} h_{i}, \quad \rho=\sum_{i=1}^{3} R_{\rho i} h_{i}$.

The rotation matrix $R$ as well as the corresponding mass matrix are given in Appendix B. $H$ and $S$ are the so-called interaction eigenstates. In particular, $H$ couples to the gauge fields in the same way that the usual MSM Higgs does.

\section{A. Integrating out the heavy Higgs fields}

In this section we want to integrate out the heavy scalars in $\Phi_{12}$ of Eq. (32) in order to build a low-energy effective theory at the $\mathrm{TeV}$ scale with an axion and a light Higgs.
As a first step, let us imagine that all the states in $\Phi_{12}$ are heavy; upon their integration we will recover the Effective Chiral Lagrangian [15]

$$
\mathcal{L}=\frac{v^{2}}{4} \operatorname{Tr} D_{\mu} \mathcal{U}^{\dagger} D^{\mu} \mathcal{U}+\sum_{i=0}^{13} a_{i} \mathcal{O}_{i}
$$

where the $\mathcal{O}_{i}$ is a set of local gauge invariant operators [16], and the symbol $D_{\mu}$ represents the covariant derivative defined in (31). The corresponding effective couplings $a_{i}$ collect the low-energy information (up to energies $E \simeq 4 \pi v$ ) pertaining to the heavy states integrated out. In the unitarity gauge, the term $D_{\mu} \mathcal{U}^{\dagger} D^{\mu} \mathcal{U}$ would generate the gauge boson masses.

If a light Higgs $\left(h=h_{1}\right)$ and axion are present, they have to be included explicitly as dynamical states [17], and the corresponding effective Lagrangian will be (gauge terms are omitted in the present discussion)

$$
\begin{aligned}
\mathcal{L}= & \frac{v^{2}}{4}\left(1+2 g_{1} \frac{h}{v}+g_{2} \frac{h^{2}}{v^{2}}+\cdots\right) \operatorname{Tr} \mathcal{D}_{\mu} \mathcal{U}^{\dagger} \mathcal{D}^{\mu} \mathcal{U} \\
& +\left(\frac{v_{\phi}^{2}}{v_{\phi}^{2}+v^{2} s_{2 \beta}}\right) \partial_{\mu} a_{\phi} \partial^{\mu} a_{\phi}+\frac{1}{2} \partial_{\mu} h \partial^{\mu} h-V(h) \\
& +\sum_{i=0}^{13} a_{i}\left(\frac{h}{v}\right) \mathcal{O}_{i}+\mathcal{L}_{\text {ren }},
\end{aligned}
$$

where ${ }^{3}$

$$
\mathcal{D}_{\mu} \mathcal{U}=D_{\mu} \mathcal{U}+\mathcal{U}\left(\partial_{\mu} U_{a}\right) U_{a}^{\dagger}
$$

formally amounting to a redefinition of the "right" gauge field and

\footnotetext{
${ }^{3}$ Note that the axion kinetic term is not well normalized in this expression yet. Extra contributions to the axion kinetic term also come from the term in the first line of Eq. (38). Only once we include these extra contributions, the axion kinetic term gets well normalized. See also discussion below.
} 


$$
\begin{aligned}
V(h)= & \frac{m_{h}^{2}}{2} h^{2}-d_{3}(\lambda v) h^{3}-d_{4} \frac{\lambda}{4} h^{4}, \\
\mathcal{L}_{\text {ren }}= & \frac{c_{1}}{v^{4}}\left(\partial_{\mu} h \partial^{\mu} h\right)^{2}+\frac{c_{2}}{v^{2}}\left(\partial_{\mu} h \partial^{\mu} h\right) \operatorname{Tr} \mathcal{D}_{\nu} \mathcal{U}^{\dagger} \mathcal{D}^{\nu} \mathcal{U} \\
& +\frac{c_{3}}{v^{2}}\left(\partial_{\mu} h \partial^{\nu} h\right) \operatorname{Tr} \mathcal{D}^{\mu} \mathcal{U}^{\dagger} \mathcal{D}_{\nu} \mathcal{U} .
\end{aligned}
$$

Here $h$ is the lightest $0^{+}$mass eigenstate, with mass $125 \mathrm{GeV}$ but couplings in principle different from the ones of a MSM Higgs. The terms in $\mathcal{L}_{\text {ren }}$ are required for renormalizability [18] at the one-loop level and play no role in the discussion.

The couplings $a_{i}$ are now functions of $h / v, a_{i}(h / v)$, which are assumed to have a regular expansion and contribute to different effective vertices. Their constant parts $a_{i}(0)$ are related to the electroweak precision parameters ("oblique corrections").

Let us see how the previous Lagrangian (38) can be derived. First, we integrate out from $\Phi_{12}=\mathcal{U} M_{12} U_{a}$ all heavy degrees of freedom, such as $H^{ \pm}$and $A_{0}$, whereas we retain $H$ and $S$ because they contain a $h_{1}$ component, namely

$$
\begin{aligned}
\Phi_{12} & =\mathcal{U} U_{a} \bar{M}_{12}, \\
\bar{M}_{12} & =\sqrt{2}\left(\begin{array}{cc}
(v+H) c_{\beta}-S s_{\beta} & 0 \\
0 & (v+H) s_{\beta}+S c_{\beta}
\end{array}\right),
\end{aligned}
$$

where $H$ and $S$ stand, respectively, for $R_{H_{1}} h_{1}$ and $R_{S 1} h_{1}$.

When the derivatives of the kinetic term of Eq. (30) act on $\bar{M}_{12}$, we get the contribution $\partial_{\mu} h \partial^{\mu} h$ in Eq. (38). Since the unitarity matrices, $\mathcal{U}$ and $U_{a}$ drop from the potential of Eq. (10) only $V(h)$ remains.

To derive the first line of Eq. (38), we can use Eqs. (39) and (42) to work out from the kinetic term of Eq. (30) the contribution

$$
\begin{aligned}
\operatorname{Tr} & \left.\mathcal{D}_{\mu} \mathcal{U} \bar{M}_{12}\right)^{\dagger} \mathcal{D}^{\mu} \mathcal{U} \bar{M}_{12} \\
& =\frac{v^{2}}{4}\left(1+2 \frac{H}{v}+\cdots\right) \operatorname{Tr} \mathcal{D}_{\mu} \mathcal{U}^{\dagger} \mathcal{D}^{\mu} \mathcal{U}+\mathcal{L}\left(a_{\phi}, h\right)
\end{aligned}
$$

Here we used that $\bar{M}_{12} \bar{M}_{12}^{\dagger}$ has a piece proportional to the identity matrix and another proportional to $\tau_{3}$ that cannot contribute to the coupling with the gauge bosons since $\operatorname{Tr} D_{\mu} \mathcal{U}^{\dagger} D^{\mu} \mathcal{U} \tau_{3}$ vanishes identically. The linear contribution in $S$ is of this type thus decoupling from the gauge sector and as a result only terms linear in $H$ survive. Using that $\left[U_{a}, \bar{M}_{12}\right]=\left[U_{a}, \tau_{3}\right]=0$, the matrix $U_{a}$ cancels out in all traces and the only remains of the axion in the low-energy action is the modification $D_{\mu} \rightarrow \mathcal{D}_{\mu}$. The resulting effective action is invariant under global transformations $\mathcal{U} \rightarrow L \mathcal{U} R^{\dagger}$ but now $R$ is an $S U(2)$ matrix only if custodial symmetry is preserved (i.e. $\tan \beta=1$ ). Otherwise, the right global symmetry group is reduced to the $U(1)$ subgroup. It commutes with $U(1)_{\mathrm{PQ}}$.
We then reproduce (38) with $g_{1}=1$. However, this is true for the field $H$ on the lhs of Eq. (43), not $h=h_{1}$ and this will reflect in a reduction in the value of the $g_{i}$ when one considers the coupling to the lightest Higgs only.

A coupling among the $S$ field, the axion and the neutral Goldstone or the neutral gauge boson survives in Eq. (43). This will be discussed in Sec. V. As for the axion kinetic term, it is reconstructed with the proper normalization from the first term in (30) together with a contribution from the 'connection' $\left(\partial_{\mu} U_{a}\right) U_{a}^{\dagger}$ in $\operatorname{Tr} \mathcal{D}_{\mu} \mathcal{U} \mathcal{D}^{\mu} \mathcal{U}$ (see Eq. (52) in the next section). There are terms involving two axions and the Higgs that are not very relevant phenomenologically at this point. This completes the derivation of the $O\left(p^{2}\right)$ terms in the effective Lagrangian.

To go beyond this tree level and to determine the lowenergy constants $a_{i}(0)$ in particular requires a one-loop integration of the heavy degrees of freedom and matching the Green's functions of the fundamental and the effective theories.

See e.g. [15,16] for a classification of all possible operators appearing up to $\mathcal{O}\left(p^{6}\right)$ that are generated in this process. The information on physics beyond the MSM is encoded in the coefficients of the effective chiral Lagrangian operators. Without including the (lightest) Higgs field $h$ (i.e. retaining only the constant term in the functions $a_{i}(h / v)$ ) and ignoring the axion, there are only two independent $\mathcal{O}\left(p^{2}\right)$ operators:

$\mathcal{L}^{2}=\frac{v^{2}}{4} \operatorname{Tr}\left(D_{\mu} \mathcal{U} D^{\mu} \mathcal{U}^{\dagger}\right)+a_{0}(0) \frac{v^{2}}{4}\left(\operatorname{Tr}\left(\tau^{3} \mathcal{U}^{\dagger} D_{\mu} \mathcal{U}\right)\right)^{2}$

The first one is universal, its coefficient being fixed by the $W$ mass. As we just saw it is flawlessly reproduced in the 2HDM at tree level after assuming that the additional degrees of freedom are heavy. Loop corrections do not modify it if $v$ is the physical Fermi scale. The other one is related to the $\rho$ parameter. In addition there are a few $\mathcal{O}\left(p^{4}\right)$ operators with their corresponding coefficients

$$
\begin{aligned}
\mathcal{L}^{4}= & \frac{1}{2} a_{1}(0) g g^{\prime} \operatorname{Tr}\left(\mathcal{U} B_{\mu \nu} \mathcal{U}^{\dagger} W^{\mu \nu}\right) \\
& -\frac{1}{4} a_{8}(0) g^{2} \operatorname{Tr}\left(\mathcal{U} \tau^{3} \mathcal{U}^{\dagger} W_{\mu \nu}\right) \operatorname{Tr}\left(\mathcal{U} \tau^{3} \mathcal{U}^{\dagger} W^{\mu \nu}\right)+\cdots
\end{aligned}
$$

In the above expression $W_{\mu \nu}$ and $B_{\mu \nu}$ are the field strength tensors associated to the $S U(2)_{L}$ and $U(1)_{Y}$ gauge fields, respectively. In this paper we shall only consider the selfenergy, or oblique, corrections, which are dominant in the 2HDM model just as they are in the MSM.

The oblique corrections are often parametrized in terms of the parameters $\varepsilon_{1}, \varepsilon_{2}$ and $\varepsilon_{3}$ introduced in [19]. In an effective theory such as the one described by the Lagrangian (44) and (45) $\varepsilon_{1}, \varepsilon_{2}$ and $\varepsilon_{3}$ receive one loop (universal) contributions from the leading $\mathcal{O}\left(p^{2}\right)$ term 
$v^{2} \operatorname{Tr}\left(D_{\mu} \mathcal{U} D^{\mu} \mathcal{U}^{\dagger}\right)$ and tree-level contributions from the $a_{i}(0)$. Thus,

$\varepsilon_{1}=2 a_{0}(0)+\cdots \quad \varepsilon_{2}=-g^{2} a_{8}(0)+\cdots$

$\varepsilon_{3}=-g^{2} a_{1}(0)+\cdots$,

where the dots symbolize the one-loop $\mathcal{O}\left(p^{2}\right)$ contributions. The latter are totally independent of the specific symmetry breaking sector. See e.g. [12] for more details.

A systematic integration of the heavy degrees of freedom, including the lightest Higgs as external legs, would provide the dependence of the low-energy coefficient functions on $h / v$, i.e. the form of the functions $a_{i}(h / v)$. However, this is of no interest to us here.

\section{HIGGS AND AXION EFFECTIVE COUPLINGS}

The coupling of $h_{1}$ can be worked out from the one of $H$, which is exactly as in the MSM, namely

$$
\begin{aligned}
& g_{1}^{\mathrm{SM}} H W_{\mu} W^{\mu} \\
& \quad=g_{1}^{\mathrm{SM}}\left(R_{H 1} h_{1}+R_{H 2} h_{2}+R_{H 3} h_{3}\right) W_{\mu} W^{\mu}
\end{aligned}
$$

where $R_{H 1}=1-\left(v / v_{\phi}\right)^{2} A_{13}^{2} / 2$ and $g_{1}^{\mathrm{SM}} \equiv 1$. With the expression of $A_{13}$ given in Appendix B,

$$
g_{1}=g_{1}^{\mathrm{SM}} \times\left(1-\frac{2 v^{2}}{v_{\phi}^{2} \lambda_{\phi}^{2}}\left(a c_{\beta}^{2}+b s_{\beta}^{2} c_{2 \beta}-c s_{2 \beta}\right)^{2}\right) .
$$

It is clear that in cases 1 to 3 the correction to the lightest Higgs couplings to the gauge bosons are very small, experimentally indistinguishable from the MSM case. In any case the correction is negative and $g_{1}<g_{1}^{\mathrm{SM}}$.

Case 4 falls in a different category. Let us remember that this case corresponds to the situation where $c \sim \lambda_{i} v^{2} / v_{\phi}^{2}$. Then the corresponding rotation matrix is effectively $2 \times 2$, with an angle $\theta$ that is given in Appendix B. Then,

$$
g_{1}=g_{1}^{\mathrm{SM}} \cos \theta .
$$

In the custodial limit, $\lambda_{1}=\lambda_{2}$ and $\tan \beta=1$, this angle vanishes exactly and $g_{1}=g_{1}^{\text {SM }}$. Otherwise, this angle could have any value. Note, however, that when $c \gg \lambda_{i} v^{2} / v_{\phi}^{2}$ then $\theta \rightarrow 0$ and the value $g_{1} \simeq g_{1}^{\mathrm{SM}}$ is recovered. This is expected as when $c$ grows case 4 moves into case 3 . Experimentally, from the LHC results we know [20] that $g_{1}=[0.67,1.25]$ at $95 \%$ C.L.

Let us now discuss the Higgs-photon-photon coupling in this type of model. Let us first consider the contribution from gauge and scalar fields in the loop. The diagrams contributing to the coupling between the lightest scalar state $h_{1}$ and photons are exactly the same ones as in a generic 2HDM, via a loop of gauge bosons and one of charged Higgses. In the DFSZ case the only change with respect to a generic $2 \mathrm{HDM}$ could be a modification in the $h_{1} W W$ (or Higgs-Goldstone bosons coupling) or in the $h_{1} H^{+} H^{+}$tree-level couplings. The former has already been discussed while the triple coupling of the lightest Higgs to two charged Higgses gets modified in the DFSZ model to

$$
\begin{aligned}
\lambda_{h_{1} H_{+} H_{-}}= & 8 v R_{H 1}\left[\left(\lambda_{1}+\lambda_{2}\right) s_{2 \beta}^{2}+4 \lambda_{3}+2 \lambda_{4}\right] \\
& +16 v s_{2 \beta} R_{S 1}\left(\lambda_{2} c_{\beta}^{2}-\lambda_{1} s_{\beta}^{2}\right) \\
& +8 v_{\phi} R_{\rho 1}\left(a s_{\beta}^{2}+b c_{\beta}^{2}-c s_{2 \beta}\right) .
\end{aligned}
$$

Note that the first line involves only constants that are already present in a generic $2 \mathrm{HDM}$, while the second one does involve the couplings $a, b$ and $c$ characteristic of the DFSZ model.

The coupling of the lightest Higgs to the up and down quarks is obtained from the Yukawa terms in Eq. (2)

$$
\begin{aligned}
\mathcal{L}\left(u, d, h_{1}\right)= & \sqrt{2} h_{1}\left[G_{1}\left(R_{H 1} c_{\beta}-R_{S 1} s_{\beta}\right) \bar{u}_{L} u_{R}\right. \\
& \left.+G_{2}\left(R_{H 1} s_{\beta}+R_{S 1} c_{\beta}\right) \bar{d}_{L} d_{R}+\text { H.c. }\right]
\end{aligned}
$$

The corresponding entries of the rotation matrix in the $0^{+}$sector can be found in Appendix B. In cases 1, 2 and 3 the relevant entries are $R_{H 1} \sim 1, R_{S 1} \sim v^{2} / v_{\phi}^{2}$ and $R_{\rho 1} \sim v / v_{\phi}$, respectively. Therefore, the second term in the first line is always negligible but the piece in the second one can give a sizeable contribution if $c$ is of $\mathcal{O}(1)$ (case 1). This case could therefore be excluded or confirmed from a precise determination of this coupling. In cases 2 and 3 this effective coupling aligns itself with a generic 2HDM but with large (typically $\sim 100 \mathrm{TeV}$ ) or moderately large (few $\mathrm{TeV}$ ) charged Higgs masses.

Case 4 is slightly different again. In this case $R_{H 1}=$ $\cos \theta$ and $R_{S 1}=\sin \theta$ but $R_{\rho 1}=0$. The situation is again similar to a generic 2HDM, now with masses that can be made relatively light, but with a mixing angle that because of the presence of the $c$ in (B9) terms may differ slightly from the 2HDM. For a review of current experimental fits in 2HDM the interested reader can see [11].

In this section we will also list the tree-level couplings of the axion to the light fields, thus completing the derivation of the effective low-energy theory. The tree-level couplings are very few actually as the axion does not appear in the potential, and they are necessarily derivative in the bosonic part. From the kinetic term we get

$$
\begin{aligned}
\mathcal{L}\left(a_{\phi}, h\right)= & \frac{2 R_{S 1}}{\sqrt{v_{\phi}^{2}+v^{2} s_{2 \beta}^{2}}} h_{1} \partial^{\mu} a_{\phi}\left(\partial_{\mu} G_{0}+m_{Z} Z_{\mu}\right) \\
& + \text { terms with } 2 \text { axions, }
\end{aligned}
$$


From the Yukawa terms (2) we also get

$$
\mathcal{L}\left(a_{\phi}, q, \bar{q}\right)=\frac{2 i}{\sqrt{v_{\phi}^{2}+v^{2} s_{2 \beta}^{2}}} a_{\phi}\left(m_{u} s_{\beta}^{2} \bar{u} \gamma_{5} u+m_{d} c_{\beta}^{2} \bar{d} \gamma_{5} d\right) .
$$

The loop-induced couplings between the axion and gauge bosons (such as the anomaly-induced coupling $a_{\phi} \tilde{F} F$, of extreme importance for direct axion detection [14]) will not be discussed here as they are amply reported in the literature.

\section{MATCHING THE DFSZ MODEL TO 2HDM}

The most general $2 \mathrm{HDM}$ potential can be read ${ }^{4}$ e.g. from $[10,11]$

$$
\begin{aligned}
V\left(\phi_{1}, \phi_{2}\right)= & m_{11}^{2} \phi_{1}^{\dagger} \phi_{1}+m_{22}^{2} \phi_{2}^{\dagger} \phi_{2}-\left[m_{12}^{2} \phi_{1}^{\dagger} \phi_{2}+\text { H.c. }\right] \\
& +\frac{\Lambda_{1}}{2}\left(\phi_{1}^{\dagger} \phi_{1}\right)^{2}+\frac{\Lambda_{2}}{2}\left(\phi_{2}^{\dagger} \phi_{2}\right)^{2} \\
& +\Lambda_{3}\left(\phi_{1}^{\dagger} \phi_{1}\right)\left(\phi_{2}^{\dagger} \phi_{2}\right)+\Lambda_{4}\left|\phi_{1}^{\dagger} \phi_{2}\right|^{2} \\
& +\left[\frac{\Lambda_{5}}{2}\left(\phi_{1}^{\dagger} \phi_{2}\right)^{2}+\Lambda_{6}\left(\phi_{1}^{\dagger} \phi_{1}\right)\left(\phi_{1}^{\dagger} \phi_{2}\right)\right. \\
& \left.+\Lambda_{7}\left(\phi_{2}^{\dagger} \phi_{2}\right)\left(\phi_{1}^{\dagger} \phi_{2}\right)+\text { H.c. }\right] .
\end{aligned}
$$

This potential contains 4 complex and 6 real parameters (i.e. 14 real numbers). The most popular $2 \mathrm{HDM}$ is obtained by imposing a $Z_{2}$ symmetry that is softly broken; namely $\Lambda_{6}=\Lambda_{7}=0$ and $m_{12} \neq 0$. The $Z_{2}$ approximate invariance helps remove flavor-changing neutral current at tree level. A special role is played by the term proportional to $m_{12}$. This term softly breaks $Z_{2}$ but is necessary to control the decoupling limit of the additional scalars in a $2 \mathrm{HDM}$ and to eventually reproduce the MSM with a light Higgs.

In the DFSZ model discussed here $v_{\phi}$ is very large and at low energies the additional singlet field $\phi$ reduces approximately to $\phi \simeq v_{\phi} \exp \left(a_{\phi} / v_{\phi}\right)$. Indeed, from (35) we see that $\phi$ has a $A_{0}$ component but it can be in practice neglected for an invisible axion since this component is $\propto v / v_{\phi}$. In addition the radial variable $\rho$ can be safely integrated out.

Thus, the low-energy effective theory defined by the DFSZ model is a particular type of 2HDM model with the nontrivial benefit of solving the strong $C P$ problem thanks to the appearance of an invisible axion. ${ }^{5}$ Indeed DFSZ reduces at low energy to a $2 \mathrm{HDM}$ containing 9 parameters

\footnotetext{
${ }^{4}$ We have relabeled $\lambda \rightarrow \Lambda$ to avoid confusion with the potential of the DFSZ model.

${ }^{5}$ Recall that mass generation due to the anomalous coupling with gluons has not been considered in this work.
}

in practice (see below, note that $v_{\phi}$ is used as input) instead of the 14 of the general $2 \mathrm{HDM}$ case.

The constants $\Lambda_{6,7}$ are absent as in many $Z_{2}$ invariant $2 \mathrm{HDM}$ but also $\Lambda_{5}=0$. All these terms are not invariant under the Peccei-Quinn symmetry. In addition the $m_{12}$ that sofly breaks $Z_{2}$ and is necessary to control the decoupling to the MSM is dynamically generated by the PQ spontaneously symmetry breaking. There is no $\mu=m_{12}$ problem here concerning the naturalness of having nonvanishing $\mu$.

At the electroweak scale the DFSZ potential of eq. (6) can be matched to the 2HDM terms of (54) by the substitutions

$$
\begin{aligned}
& m_{11}^{2}=\left[-2 \lambda_{1} V_{1}^{2}+2 \lambda_{3}\left(V_{1}^{2}+V_{2}^{2}\right)+a v_{\phi}^{2}\right] / 4 \\
& m_{22}^{2}=\left[-2 \lambda_{1} V_{2}^{2}+2 \lambda_{3}\left(V_{1}^{2}+V_{2}^{2}\right)+b v_{\phi}^{2}\right] / 4 \\
& m_{12}^{2}=c v_{\phi}^{2} / 4 \\
& \Lambda_{1}=\left(\lambda_{1}+\lambda_{3}\right) / 8, \quad \Lambda_{2}=\left(\lambda_{2}+\lambda_{3}\right) / 8 \\
& \Lambda_{3}=\left(2 \lambda_{3}+\lambda_{4}\right) / 16, \quad \Lambda_{4}=-\lambda_{4} / 16, \\
& \Lambda_{5}=0, \quad \Lambda_{6}=0, \quad \Lambda_{7}=0 .
\end{aligned}
$$

Combinations of parameters of the DFSZ potential can be determined from the four masses $m_{h_{1}}, m_{h_{2}}, m_{A_{0}}$ and $m_{H^{+}}$ and the two parameters $g_{1}($ or $\theta)$ and $\lambda_{h_{1} H_{+} H_{-}}$that controls the Higgs- $W W$ and (indirectly) the Higgs- $\gamma \gamma$ couplings, whose expression in terms of the parameters of the potential has been given. As we have seen for generic couplings, all masses but the lightest Higgs decouple and the effective couplings take their MSM values. In the phenomenologically more interesting cases (cases 3 and 4) two of the remaining constants $(a, b)$ drop in practice from the lowenergy predictions and the effective 2HDM corresponding to DFSZ depends only on seven parameters. If in addition custodial symmetry is assumed to be exact or nearly exact, the relevant parameters are actually totally determined by measuring three masses and the two couplings ( $m_{h_{2}}$ turns out to be equal to $m_{H^{+}}$if custodial invariance holds). Therefore LHC has the potential of fully determining all the relevant parameters of the DFSZ model.

Eventually the LHC and perhaps a LC will be hopefully able to assess the parameters of the $2 \mathrm{HDM}$ potential and their symmetries to check the DFSZ relations. Of course finding a pattern of couplings in concordance with the pattern predicted by the low-energy limit of DFSZ model would not yet prove the latter to be the correct microscopic theory as this would require measuring the axion couplings, which are not present in a 2HDM. In any case, it should be obvious that the effective theory of the DFSZ is significantly more restrictive than a general 2HDM.

We emphasize that the above discussion refers mostly to case 4 as discussed in this work and it partly applies to case 
3 too. Cases 1 and 2 are in practice indistinguishable from the MSM up to energies that are substantially larger from the ones currently accessible, apart from the presence of the axion itself. As we have seen, the DFSZ in this case is quite predictive and it does not correspond to a generic $2 \mathrm{HDM}$ but to one where massive scalars are all decoupled with the exception of the $125 \mathrm{GeV}$ Higgs.

\section{CONSTRAINTS FROM ELECTROWEAK PARAMETERS}

For the purposes of setting bounds on the masses of the new scalars in the $2 \mathrm{HDM}, \varepsilon_{1}=\Delta \rho$ is the most effective one. For this reason we will postpone the analysis of $\varepsilon_{2}$ and $\varepsilon_{3}$ to a future publication.

$\varepsilon_{1}$ can be computed by [19]

$$
\varepsilon_{1} \equiv \frac{\Pi_{W W}(0)}{M_{W}^{2}}-\frac{\Pi_{Z Z}(0)}{M_{Z}^{2}}
$$

with the gauge boson vacuum polarization functions defined as

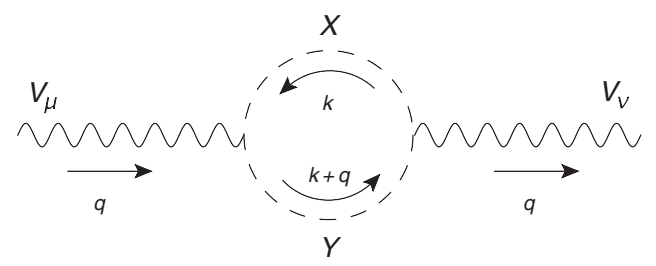

FIG. 2. Feynman diagram relevant for $\Pi_{V V}^{\mu \nu}(q)$.

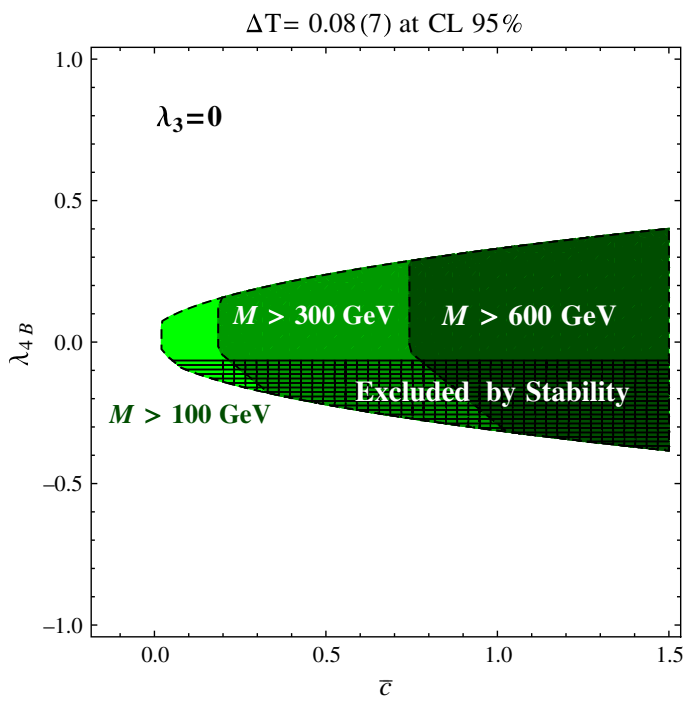

$\Pi_{V V}^{\mu \nu}(q)=g^{\mu \nu} \Pi_{V V}\left(q^{2}\right)+q^{\mu} q^{\nu}$ terms $\quad(V=W, Z)$.

We need to compute loops of the Fig. 2 type. These diagrams produce three kinds of terms. The terms proportional to two powers of the external momentum, $q_{\mu} q_{\nu}$, do not enter in $\Pi_{V V}\left(q^{2}\right)$. The terms proportional to just one power vanish upon integration. Only the terms proportional to $k_{\mu} k_{\nu}$ survive and contribute.

Although it is an unessential approximation, to keep formulae relatively simple we will compute $\varepsilon_{1}$ in the approximation $g^{\prime}=0$. The term proportional to $\left(g^{\prime}\right)^{2}$ is actually the largest contribution in the MSM (leaving aside the breaking due to the Yukawa couplings) but it is only logarithmically dependent on the masses of any putative scalar state and it can be safely omitted for our purposes [12]. The underlying reason is that in the 2HDM custodial symmetry is "optional" in the scalar sector and it is natural to investigate powerlike contributions that would provide the strongest constraints. We obtain, in terms of the mass eigenstates and the rotation matrix of Eq. (36),

$$
\begin{aligned}
\varepsilon_{1}= & \frac{1}{16 \pi^{2} v^{2}}\left[m_{H_{ \pm}}^{2}-\frac{v_{\phi}^{2}}{v_{\phi}^{2}+v^{2} s_{2 \beta}^{2}} f\left(m_{H_{ \pm}}^{2}, m_{A_{0}}^{2}\right)\right. \\
& \left.+\sum_{i=1}^{3} R_{S i}^{2}\left(\frac{v_{\phi}^{2}}{v_{\phi}^{2}+v^{2} s_{2 \beta}^{2}} f\left(m_{A_{0}}^{2}, m_{h_{i}}^{2}\right)-f\left(m_{H_{ \pm}}^{2}, m_{h_{i}}^{2}\right)\right)\right],
\end{aligned}
$$

where $f(a, b)=a b /(b-a) \log b / a$ and $f(a, a)=a$. Setting $v_{\phi} \rightarrow \infty$ and keeping Higgs masses fixed, we

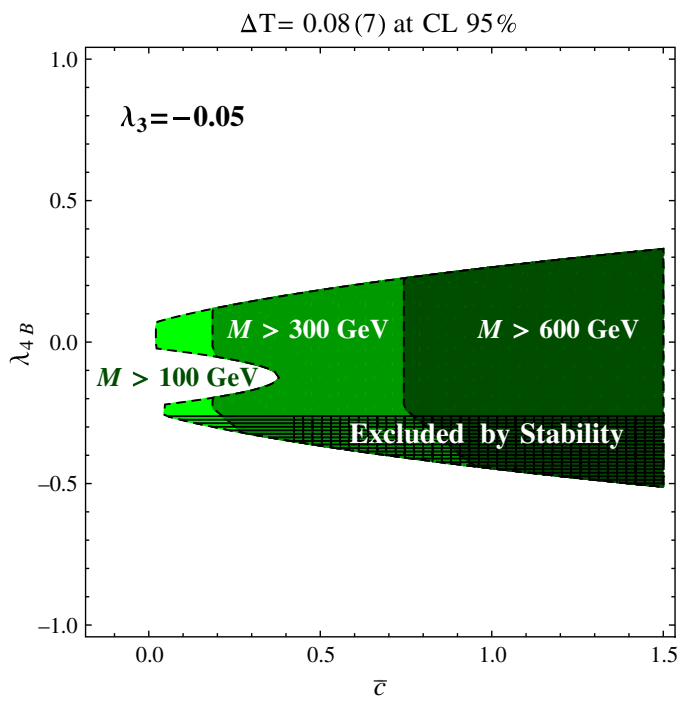

FIG. 3 (color online). Exclusion region for a "quasicustodial" 2 HDM potential with a $\lambda_{4 B}=\lambda_{4}-2 \lambda$ custodial breaking term. In this limit, the masses depend on the free parameters $\lambda_{4 B}=\lambda_{4}-2 \lambda, \lambda_{3}$ and $\bar{c}=c v_{\phi}^{2} / v^{2}$, and then the vacuum stability conditions of Appendix D and $\Delta T$ can be used to exclude regions of the free parameters above. The left, right plots are for $\lambda_{3}=-0.05, \lambda_{3}=0$, respectively. Different color regions imply different cuts assuming that all masses $\left(m_{A_{0}}, m_{H_{ \pm}}\right.$and $\left.m_{h_{2}}\right)$ are greater than 100,300 or $600 \mathrm{GeV}$ (light to dark). The potential becomes unstable for $\lambda_{3}>0.03$. 

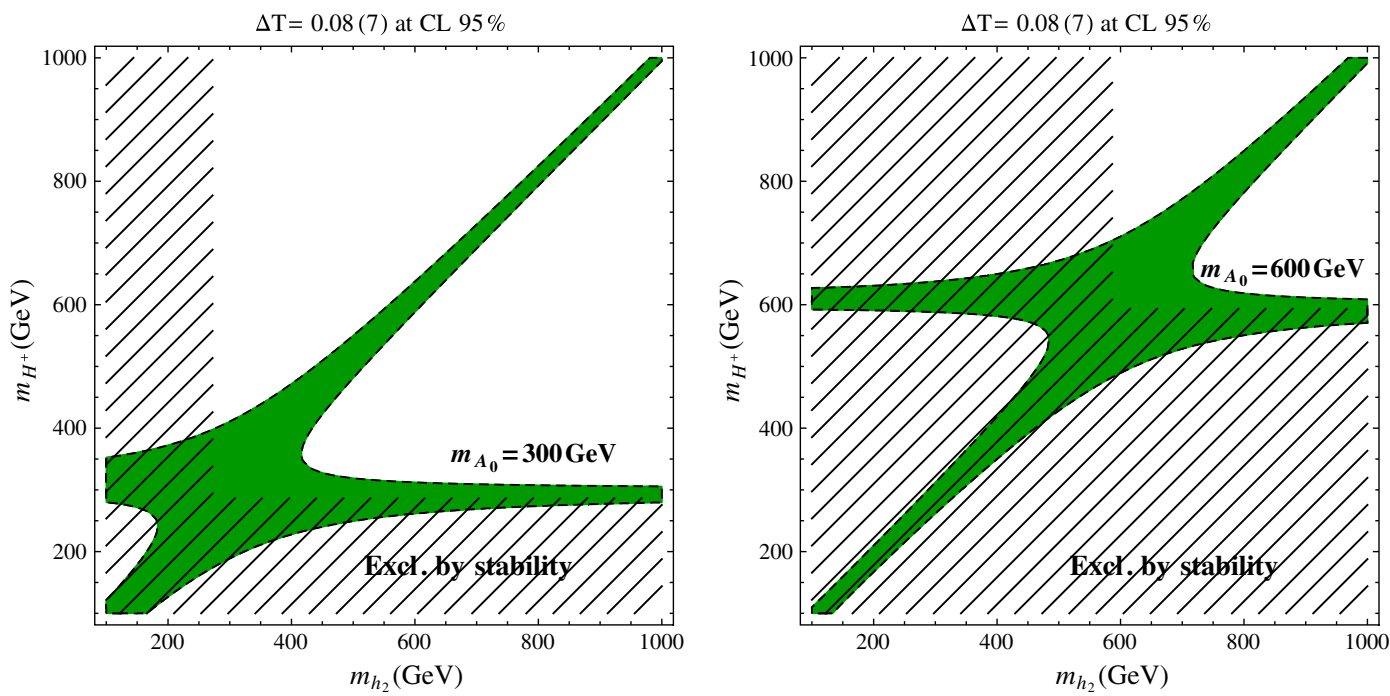

FIG. 4 (color online). Exclusion plot imposed by the constraint from $\Delta T$ on the second $0^{+}$state (i.e. "second Higgs") and the charged Higgs masses for two reference values of $M_{A_{0}}$ (left: $300 \mathrm{GeV}$, right: $600 \mathrm{GeV}$ ) in the "quasicustodial" case discussed in Fig. 3. The concentration of points along approximately two axis is easy to understand after inspection of the relevant formula for $\Delta T$. $\Delta T$ is vanishing at the custodial limit $M_{h_{2}}=M_{H^{ \pm}}$, and also for $M_{H^{ \pm}}=M_{A_{0}}$. The regions excluded by considerations of stability of the potential are shown.

formally recover the $\Delta \rho$ expression in the $2 \mathrm{HDM}$ (see the Appendix in [12]), namely

$$
\begin{aligned}
\varepsilon_{1}= & \frac{1}{16 \pi^{2} v^{2}}\left[m_{H_{ \pm}}^{2}-f\left(m_{H_{ \pm}}^{2}, m_{A_{0}}^{2}\right)\right. \\
& \left.+\sum_{i=1}^{3} R_{S i}^{2}\left(f\left(m_{A_{0}}^{2}, m_{h_{i}}^{2}\right)-f\left(m_{H_{ \pm}}^{2}, m_{h_{i}}^{2}\right)\right)\right] .
\end{aligned}
$$

Now, in the limit $v_{\phi} \rightarrow \infty$ and $m_{H_{+}} \rightarrow m_{A_{0}}$ (cases 1,2 or 3 previously discussed) the $\Delta \rho$ above will go to zero as

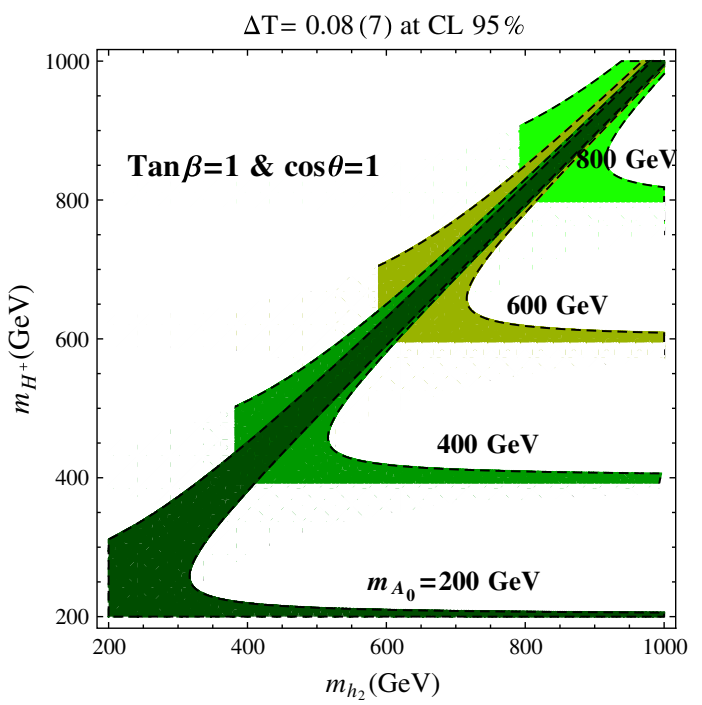

$v / v_{\phi}$ at least and the experimental bound is fulfilled automatically.

However, we are particularly interested in case 4 that allows for a light spectrum of new scalar states. We will study this in two steps. First we assume a "quasicustodial" setting whereby we assume that custodial symmetry is broken only via the coupling $\lambda_{4 B}=\lambda_{4}-2 \lambda$ being nonzero. Imposing vacuum stability (see e.g. Appendix D) and the experimental bound of $\left(\varepsilon_{1}-\varepsilon_{1}^{\mathrm{SM}}\right) / \alpha=\Delta T=0.08(7)$ from the electroweak fits in [21] one gets the exclusion plots shown in Fig. 3.

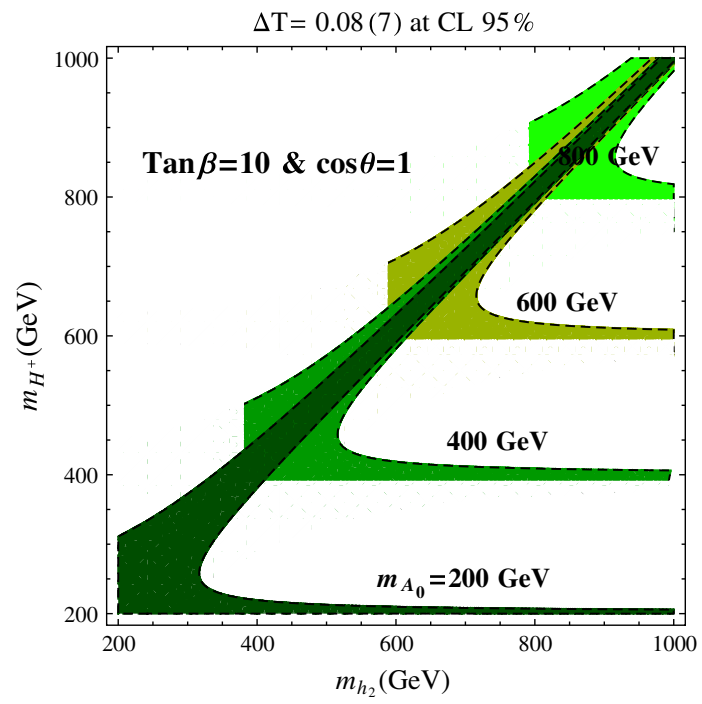

FIG. 5 (color online). Exclusion plot imposed by the constraint from $\Delta T$ on the second $0^{+}$state (i.e. 'second Higgs') and the charged Higgs masses for several reference values of $m_{A_{0}}$ and $\tan \beta$ in the general case. The value $\cos \theta=1$ is assumed here. The successive horizontal bands correspond to different values of $m_{A_{0}}$. The stability bounds have already been implemented, effectively cutting off the left and lower arms of the regions otherwise acceptable. 


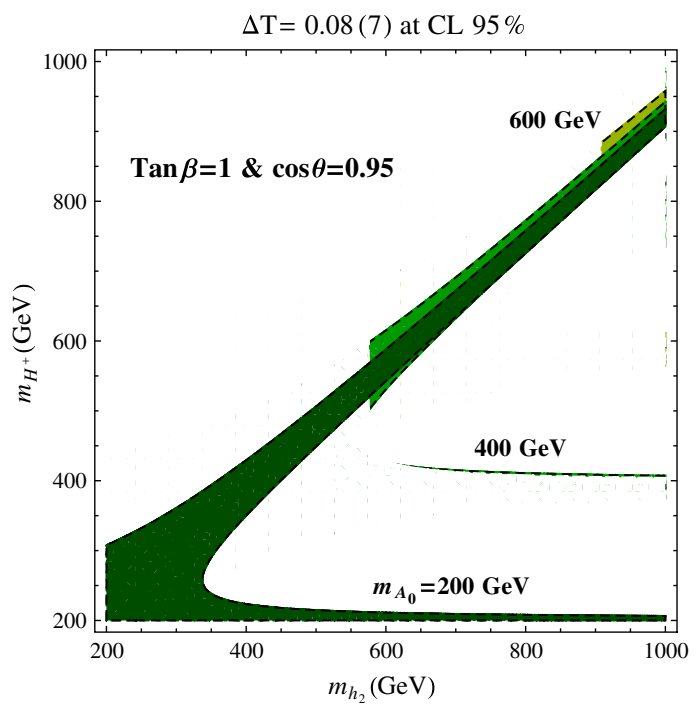

FIG. 6 (color online). Exclusion plot imposed by the constraint from $\Delta T$ on the second $0^{+}$state (i.e. 'second Higgs') and the charged Higgs masses for several reference values of $m_{A_{0}}$. Here we take $\tan \beta=1$ and allow $\cos \theta=0.95$, which is consistent with present constraints.

It is also interesting to show (in this same "quasicustodial" limit) the range of masses allowed by the present constraints on $\Delta T$, without any reference to the parameters in the potential. This is shown for two reference values of $m_{A_{0}}$ in Fig. 4. Note the severe constraints due to the requirement of vacuum stability.

Finally let us turn to the consideration of the general case 4 . We now completely give up custodial symmetry and hence the three masses $m_{A_{0}}, m_{H_{ \pm}}$and $m_{h_{2}}$ are unrelated, except for the eventual lack of stability of the potential. In this case, the rotation $R$ can be different form the identity which was the case in the "quasicustodial" scenario above. In particular, $R_{S 2}=\cos \theta$ from Appendix $\mathrm{B}$ and the angle $\theta$ is not vanishing. However, experimentally $\cos \theta$ is known to be very close to one (see Sec. V). If we assume that $\cos \theta$ is exactly equal to one, we get the exclusion/acceptance regions shown in Fig. 5. Finally, Fig. 6 depicts the analogous plot for $\cos \theta=0.95$ that is still allowed by existing constraints. We wee that the allowed range of masses are much more severely restricted in this case.

\section{CONCLUSIONS}

With the LHC experiments gathering more data, the exploration of the symmetry breaking sector of the Standard Model will gain renewed impetus. Likewise, it is important to search for dark matter candidates as this is a degree of freedom certainly missing in the minimal Standard Model. An invisible axion is an interesting candidate for dark matter; however, trying to look for direct evidence of its existence at the LHC is hopeless as it is extremely weakly coupled. Therefore we have to resort to less direct ways to explore this sector by formulating consistent models that include the axion and deriving consequences that could be experimentally tested.

In this work we have explored such consequences in the DFSZ model, an extension of the popular 2HDM. A necessary characteristic of models with an invisible axion is the presence of the Peccei-Quinn symmetry. This restricts the form of the effective potential. We have taken into account the recent data on the Higgs mass and several effective couplings, and included the constraints from electroweak precision parameters.

Four possible scenarios have been considered. In virtually all points of parameter space of the DFSZ model we do not really expect to see any relevant modifications with respect to the minimal Standard Model predictions. The new scalars have masses of order $v_{\phi}$ or $\sqrt{v v_{\phi}}$ in two of the cases discussed. The latter could perhaps be reachable with a $100 \mathrm{TeV}$ circular collider although this is not totally guaranteed. In a third case, it would be possible to get scalars in the multi-TeV region, making this case testable in the future at the LHC. Finally, we have identified a fourth situation where a relatively light spectrum emerges. The last two cases correspond to a situation where the coupling between the singlet and the two doublets is of order $v^{2} / v_{\phi}^{2}$; i.e. very small $\left(10^{-10}\right.$ or less $)$ and in order to get a relatively light spectrum in addition one has to require some couplings to be commensurate (but not necessarily fine-tuned).

The fact that some specific couplings are required to be very small may seem odd, but as it has been argued elsewhere it is technically natural, as the couplings in question do break some extended symmetry and are therefore protected. From this point of view these values are perfectly acceptable.

The results on the scalar spectrum are derived here at tree level only and are of course subject to large radiative corrections in principle. However, one should note two ingredients that should ameliorate the hierarchy problem. The first observation is that the mass of the $0^{-}$scalar is directly proportional to $c$; it is exactly zero if the additional symmetries discussed in [7] hold. It is therefore somehow protected. On the other hand, custodial symmetry relates different masses, helping to maintain other relations. Some hierarchy problem should still remain but of a magnitude very similar to the one already present in the minimal Standard Model.

We have imposed on the model known constraints such as the fulfilment of the bounds on the $\rho$-parameter. These bounds turn out to be automatically fulfilled in most of parameter space and become only relevant when the spectrum is light (case 4). This is particularly relevant as custodial symmetry is by no means automatic in the 2HDM. Somehow the introduction of the axion and the related Peccei-Quinn symmetry makes possible custodially 
violating consequences naturally small. We have also considered the experimental bounds on the Higgs-gauge bosons and Higgs-two photons couplings. Together with four scalar masses, these parameters determine in an almost unique way the DFSZ potential, thus showing that it has subtantial less room to maneuver than a generic 2HDM.

In conclusion, DFSZ models containing an invisible axion are natural and, in spite of the large scale that appears in the model to make the axion nearly invisible, there is the possibility that they lead to a spectrum that can be tested at the LHC. This spectrum is severely constrained, making it easier to prove or disprove such possibility in the near future. On the other hand, it is perhaps more likely that the new states predicted by the model lie beyond the LHC range. In this situation the model hides itself by making indirect contributions to most observables quite small.

\section{ACKNOWLEDGMENTS}

This work is supported by Spanish Ministry of Economy and Competitiveness Grant No. FPA2013-46570 and Generalitat de Catalunya Grant No. 2014-SGR-104 and Consolider Grant No. CSD2007-00042 (CPAN). A. R. acknowledges the financial support of a FPU predoctoral grant.

\section{APPENDIX A: MINIMIZATION CONDITIONS OF THE POTENTIAL}

The minimization conditions for the potential (6) are

$$
\begin{gathered}
\lambda_{1}\left(2 v^{2} c_{\beta}^{2}-V_{1}^{2}\right)+\lambda_{3}\left(2 v^{2}-V_{1}^{2}-V_{2}^{2}\right) \\
+\frac{v_{\phi}^{2}}{2}(a+c \tan \beta)=0, \\
\lambda_{2}\left(2 v^{2} s_{\beta}^{2}-V_{2}^{2}\right)+\lambda_{3}\left(2 v^{2}-V_{1}^{2}-V_{2}^{2}\right) \\
\quad+\frac{v_{\phi}^{2}}{2}\left(b+\frac{c}{\tan \beta}\right)=0, \\
\lambda_{\phi}\left(v_{\phi}^{2}-V_{\phi}^{2}\right)+2 v^{2}\left(a c_{\beta}^{2}+b s_{\beta}^{2}-c s_{2 \beta}\right)=0 .
\end{gathered}
$$

These allow us to eliminate the dimensionful parameters $V_{\phi}, V_{1}$ and $V_{2}$ in favor of the different couplings, $v$ and $v_{\phi}$. In the case where $\lambda_{\phi}=0$, it is also possible to eliminate $c$.

\section{APPENDIX B: $0^{+}$NEUTRAL SECTOR MASS MATRIX}

The $3 \times 3$ mass matrix is

$$
M_{H S \rho}=4\left(\begin{array}{ccc}
8 v^{2}\left(\lambda_{1} c_{\beta}^{4}+\lambda_{2} s_{\beta}^{4}+\lambda_{3}\right) & 4 v^{2}\left(-\lambda_{1} c_{\beta}^{2}+\lambda_{2} s_{\beta}^{2}\right) s_{2 \beta} & 2 v v_{\phi}\left(a c_{\beta}^{2}+b s_{\beta}^{2}-c s_{2 \beta}\right) \\
4 v^{2}\left(-\lambda_{1} c_{\beta}^{2}+\lambda_{2} s_{\beta}^{2}\right) s_{2 \beta} & \frac{2 c v_{\phi}^{2}}{s_{2 \beta}}+2 v^{2}\left(\lambda_{1}+\lambda_{2}\right) s_{2 \beta}^{2} & -v v_{\phi}\left[(a-b) s_{2 \beta}+2 c c_{2 \beta}\right] \\
2 v v_{\phi}\left(a c_{\beta}^{2}+b s_{\beta}^{2}-c s_{2 \beta}\right) & -v v_{\phi}\left[(a-b) s_{2 \beta}+2 c c_{2 \beta}\right] & \lambda_{\phi} v_{\phi}^{2}
\end{array}\right) \text {. }
$$

This is diagonalized with a rotation

$$
\left(\begin{array}{c}
H \\
S \\
\rho
\end{array}\right)=R\left(\begin{array}{l}
h_{1} \\
h_{2} \\
h_{3}
\end{array}\right) .
$$

We write the rotation matrix as

$R=\exp \left(\frac{v}{v_{\phi}} A+\frac{v^{2}}{v_{\phi}^{2}} B\right), \quad A^{T}=-A, \quad B^{T}=-B$

and work up to second order in $v / v_{\phi}$. We find

$$
A_{12}=B_{13}=B_{23}=0
$$

so the matrix is

$$
R=\left(\begin{array}{ccc}
1-\frac{v^{2}}{v_{\phi}^{2}} \frac{A_{13}^{2}}{2} & -\frac{v^{2}}{v_{\phi}^{2}} \frac{A_{13} A_{23}-2 B_{12}}{2} & \frac{v}{v_{\phi}} A_{13} \\
-\frac{v^{2}}{v_{\phi}^{2}} \frac{A_{13} A_{23}+2 B_{12}}{2} & 1-\frac{v^{2} A_{23}^{2}}{v_{\phi}^{2}} & \frac{v}{v_{\phi}} A_{23} \\
-\frac{v}{v_{\phi}} A_{13} & -\frac{v}{v_{\phi}} A_{23} & 1-\frac{v^{2}}{v_{\phi}^{2}} \frac{A_{13}^{2}+A_{23}^{2}}{2}
\end{array}\right),
$$

with

$$
\begin{aligned}
& A_{13}=\frac{2}{\lambda_{\phi}}\left(a c_{\beta}^{2}+b s_{\beta}^{2}-c s_{2 \beta}\right), \\
& A_{23}=\frac{(a-b) s_{2 \beta}+2 c c_{2 \beta}}{\frac{2 c}{s_{2 \beta}}-\lambda_{\phi}},
\end{aligned}
$$

$$
\begin{aligned}
B_{12}= & -\frac{2}{c} s_{2 \beta}^{2}\left(\lambda_{1} c_{\beta}^{2}-\lambda_{2} s_{\beta}^{2}\right) \\
& +\frac{s_{2 \beta}}{\lambda_{\phi} c} \frac{c-\lambda_{\phi} s_{2 \beta}}{2 c-\lambda_{\phi} s_{2 \beta}}\left(a c_{\beta}^{2}+b s_{\beta}^{2}-c s_{2 \beta}\right) \\
& \times\left[(a-b) s_{2 \beta}+2 c c_{2 \beta}\right] .
\end{aligned}
$$


In the case of Sec. III when the breaking of custodial symmetry is $S U(2) \times S U(2) \rightarrow U(1)$ the mass matrix is

$$
M_{H S \rho}=4\left(\begin{array}{ccc}
8 v^{2}\left[\lambda_{3}+\lambda\left(s_{\beta}^{4}+c_{\beta}^{4}\right)\right] & -2 \lambda v^{2} s_{4 \beta} & 2 v v_{\phi}\left(a+2 \lambda \frac{v^{2}}{v_{\phi}^{2}} s_{2 \beta}^{2}\right) \\
-2 \lambda v^{2} s_{4 \beta} & -4 \lambda v^{2} c_{2 \beta}^{2} & 2 \lambda \frac{v^{3}}{v_{\phi}} s_{4 \beta} \\
2 v v_{\phi}\left(a+2 \lambda \frac{v^{2}}{v_{\phi}^{2}} s_{2 \beta}^{2}\right) & 2 \lambda \frac{v^{3}}{v_{\phi}} s_{4 \beta} & \lambda v_{\phi}^{2}
\end{array}\right) .
$$

For case 4 of Sec. III the rotation matrix is

$$
R=\left(\begin{array}{ccc}
\cos \theta & -\sin \theta & 0 \\
\sin \theta & \cos \theta & 0 \\
0 & 0 & 1
\end{array}\right)
$$

$\tan 2 \theta=-\frac{\left(\lambda_{1} c_{\beta}^{2}-\lambda_{2} s_{\beta}^{2}\right) s_{2 \beta}}{\left(\lambda_{1} c_{\beta}^{2}-\lambda_{2} s_{\beta}^{2}\right) c_{2 \beta}+\lambda_{3}-c v_{\phi}^{2} /\left(4 v^{2} s_{2 \beta}\right)}$.

\section{APPENDIX C: THE LIMIT $\lambda_{\phi}=0$}

The eigenvalues of the $3 \times 3$ mass matrix in the $0^{+}$sector are

$$
\begin{aligned}
& m_{h_{1}}^{2}=32 v^{2}\left(\lambda_{1} c_{\beta}^{4}+\lambda_{2} s_{\beta}^{4}+\lambda_{3}\right) \\
& m_{h_{2}}^{2}=\frac{v_{\phi}^{2}}{2} s_{2 \beta}^{2}\left(a c_{\beta}^{2}+b s_{\beta}^{2}\right)+\mathcal{O}\left(v^{2}\right) \\
& m_{h_{3}}^{2}=-8 v^{2} \frac{\left(a c_{\beta}-b s_{\beta}\right)^{2}}{a c_{\beta}^{2}+b s_{\beta}^{2}}
\end{aligned}
$$

Either $m_{2}^{2}$ or $m_{3}^{2}$ is negative. Note that the limit of $a, b$ small cannot be taken directly in this case.

\section{APPENDIX D: VACUUM STABILITY CONDITIONS AND MASS RELATIONS}

Vacuum stability implies the following conditions on the parameters of the potential [10]:

$$
\begin{aligned}
\lambda_{1}+\lambda_{3} & >0, \\
\lambda_{2}+\lambda_{3} & >0, \\
2 \lambda_{3}+\lambda_{4}+2 \sqrt{\left(\lambda_{1}+\lambda_{3}\right)\left(\lambda_{2}+\lambda_{3}\right)} & >0, \\
\lambda_{3}+\sqrt{\left(\lambda_{1}+\lambda_{3}\right)\left(\lambda_{2}+\lambda_{3}\right)} & >0 .
\end{aligned}
$$

In the case of custodial symmetry except for $\lambda_{4 B} \neq 0$, these conditions reduce to

$$
\lambda+\lambda_{3}>0, \quad \lambda+2 \lambda_{3}>0, \quad 4 \lambda+4 \lambda_{3}+\lambda_{4 B}>0
$$

and assuming $a, b, c$ very small (e.g. case 4 ) they impose two conditions on the masses for

$$
m_{A_{0}}^{2}+m_{h_{1}}^{2}-m_{h_{2}}^{2}>0, \quad m_{H_{ \pm}}^{2}+m_{h_{1}}^{2}-m_{A_{0}}^{2}>0 .
$$

\section{APPENDIX E: VERTICES AND FEYNMAN RULES IN THE DFSZ MODEL}

In the limit of $g^{\prime}=0$, all the diagrams involved in the calculation of $\varepsilon_{1}$ are of the Fig. 2 type. All the relevant vertices are of the type seen in Fig. 7, with all momenta assumed to be incoming. The relevant Feynman rules are as follows:

\begin{tabular}{lc} 
Interaction term & Feynman rule for the vertex \\
\hline$\lambda V^{\alpha} X \partial_{\alpha} Y$ & $\lambda p_{Y}^{\mu}$ \\
$\lambda V^{\alpha} X \stackrel{\leftrightarrow}{\partial}_{\alpha} Y$ & $\lambda\left(p_{Y}-p_{X}\right)^{\mu}$
\end{tabular}

To compute $\Pi_{Z Z}$ entering Eq. (61), we need diagrams like Fig. 2 with $V=Z$. The $X, Y$ pairs are

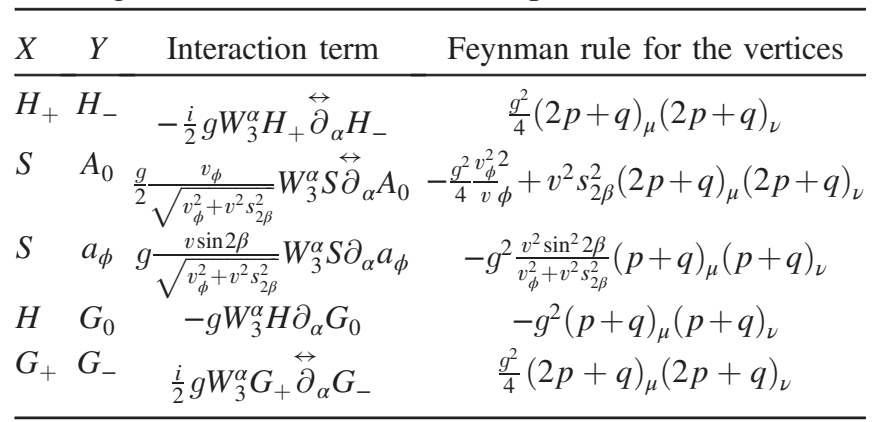

To compute $\Pi_{W W}$ entering Eq. (61), we need diagrams like Fig. 2 with $V=W_{+}$. The $X, Y$ pairs are

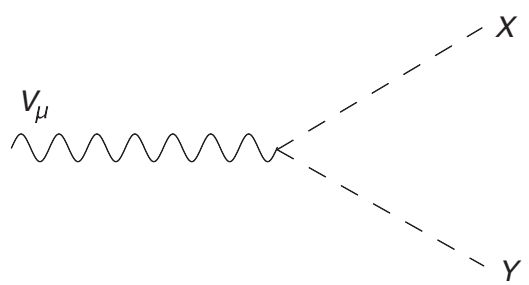

FIG. 7. Two scalars and a gauge boson. 


\begin{tabular}{lccr}
\hline$X$ & $Y$ & Interaction term & Feynman rule for the vertices \\
\hline$H_{+}$ & $S$ & $\frac{i}{2} g W_{+}^{\alpha} H_{-} \stackrel{\leftrightarrow}{\partial}_{\alpha} S$ & $\frac{g^{2}}{4}(2 p+q)_{\mu}(2 p+q)_{\nu}$ \\
$H_{+}$ & $A_{0}$ & $\frac{g}{2} \frac{v_{\phi}}{\sqrt{v_{\phi}^{2}+v^{2} s_{2 \beta}^{2}}} W_{+}^{\alpha} A_{0} \stackrel{\leftrightarrow}{\partial}_{\alpha} H_{-}-$ & $-\frac{g^{2} v_{\phi}^{2}}{4}+v^{2} s_{2 \beta}^{2}(2 p+q)_{\mu}(2 p+q)_{\nu}$ \\
$H_{+}$ & $a_{\phi}$ & $-g \frac{v \sin 2 \beta}{\sqrt{v_{\phi}^{2}+v^{2} s_{2 \beta}^{2}}} W_{+}^{\alpha} H_{-} \partial_{\alpha} a_{\phi}$ & $-g^{2} \frac{v^{2} \sin ^{2} 2 \beta}{v_{\phi}^{2}+v^{2} s_{2 \beta}^{2}}(p+q)_{\mu}(p+q)_{\nu}$ \\
$H$ & $G_{+}$ & $-g W_{+}^{\alpha} H \partial_{\alpha} G_{-}$ & $-g^{2}(p+q)_{\mu}(p+q)_{\nu}$ \\
$G_{+}$ & $G_{0}$ & $\frac{i}{2} g W_{+}^{\alpha} G_{0} \stackrel{\leftrightarrow}{\partial}_{\alpha} G_{-}$ & $\frac{g^{2}}{4}(2 p+q)_{\mu}(2 p+q)_{\nu}$ \\
\hline
\end{tabular}

[1] R. D. Peccei and H. R. Quinn, Phys. Rev. Lett. 38, 1440 (1977); S. Weinberg, Phys. Rev. Lett. 40, 223 (1978); F. Wilczek, Phys. Rev. Lett. 40, 279 (1978).

[2] L. Abbott and P. Sikivie, Phys. Lett. 120B, 133 (1983); M. Dine and W. Fischler, Phys. Lett. 120B, 137 (1983); J. Preskill, M. B. Wise, and F. Wilczek, Phys. Lett. 120B, 127 (1983).

[3] M. Kuster, G. Raffelt, and B. Beltran, Lect. Notes Phys. 741 (2008).

[4] M. Dine, W. Fischler, and M. Srednicki, Phys. Lett. 104B, 199 (1981).

[5] A. R. Zhitnitsky, Sov. J. Nucl. Phys. 31, 260 (1980); J. E. Kim, Phys. Rev. Lett. 43, 103 (1979); M. A. Shifman, A. I. Vainshtein, and V. I. Zakharov, Nucl. Phys. B166, 493 (1980).

[6] J. M. Frere, J. A. M. Vermaseren, and M. B. Gavela, Phys. Lett. 103B, 129 (1981); L. J. Hall and M. B. Wise, Nucl. Phys. B187, 397 (1981); H. Georgi, D. B. Kaplan, and L. Randall, Phys. Lett. 169B, 73 (1986); A. Celis, J. FuentesMartin, and H. Serodio, Phys. Lett. B 737, 185 (2014).

[7] R. R. Volkas, A. J. Davies, and G. C. Joshi, Phys. Lett. B 215, 133 (1988); R. Foot, A. Kobakhidze, K. L. McDonald, and R. R. Volkas, Phys. Rev. D 89, 115018 (2014).

[8] K. Allison, C. T. Hill, and G. G. Ross, Nucl. Phys. B891, 613 (2015).

[9] G. C. Branco, P. M. Ferreira, L. Lavoura, M. N. Rebelo, M. Sher, and J. P. Silva, Phys. Rep. 516, 1 (2012); F. J. Botella, G. C. Branco, A. Carmona, M. Nebot, L. Pedro, and M. N. Rebelo, J. High Energy Phys. 07 (2014) 078; S. Bertolini, L. Di Luzio, H. Kolesova, and M. Malinske, Phys. Rev. D 91, 055014 (2015).

[10] J. F. Gunion and H. E. Haber, Phys. Rev. D 67, 075019 (2003).

[11] D. Lopez-Val, T. Plehn, and M. Rauch, J. High Energy Phys. 10 (2013) 134; D. Lopez-Val and J. Sola, Phys. Rev. D 81, 033003 (2010); X. Q. Li, J. Lu, and A. Pich, J. High Energy Phys. 06 (2014) 022; A. Celis, V. Ilisie, and A. Pich, J. High Energy Phys. 12 (2013) 095; , 07 (2013) 053.

[12] P. Ciafaloni and D. Espriu, Phys. Rev. D 56, 1752 (1997).

[13] A. Pomarol and R. Vega, Nucl. Phys. B413, 3 (1994); B. Grzadkowski, M. Maniatis, and J. Wudka, J. High Energy Phys. 11 (2011) 030.

[14] J. Beringer et al. (Particle Data Group), Phys. Rev. D 86, 010001 (2012); A. H. Córsico, L. G. Althaus, A. D. Romero, A. S. Mukadam, E. García-Berro, J. Isern,
S. O. Kepler, and M. A. Corti, J. Cosmol. Astropart. Phys. 12 (2012) 010; E. Arik et al. (CAST Collaboration), J. Cosmol. Astropart. Phys. 02 (2009) 008; I. G. Irastorza et al. (IAXO Collaboration), J. Cosmol. Astropart. Phys. 06 (2011) 013; R. Bähre et al. (ALPS Collaboration), JINST 09, T09001 (2013); S. J. Asztalos et al. (ADMX Collaboration), Nucl. Instrum. Methods Phys. Res., Sect. A 656, 39 (2011).

[15] A. C. Longhitano, Nucl. Phys. B188, 118 (1981); Phys. Rev. D 22, 1166 (1980); A. Dobado, D. Espriu, and M. J. Herrero, Phys. Lett. B 255, 405 (1991); D. Espriu and M. J. Herrero, Nucl. Phys. B373, 117 (1992); M. J. Herrero and E. RuizMorales, Nucl. Phys. B418, 431 (1994); B437, 319 (1995); D. Espriu and J. Matias, Phys. Lett. B 341, 332 (1995).

[16] D. Espriu and B. Yencho, Phys. Rev. D 87, 055017 (2013).

[17] G. F. Giudice, C. Grojean, A. Pomarol, and R. Rattazzi, J. High Energy Phys. 06 (2007) 045; R. Contino, M. Ghezzi, C. Grojean, M. Muhlleitner, and M. Spira, J. High Energy Phys. 07 (2013) 035; R. Alonso, M. B. Gavela, L. Merlo, S. Rigolin, and J. Yepes, Phys. Lett. B 722, 330 (2013); R. Alonso, I. Brivio, B. Gavela, L. Merlo, and S. Rigolin, J. High Energy Phys. 12 (2014) 034; G. Buchalla, O. Catà, and C. Krause, Nucl. Phys. B880, 552 (2014); B894, 602 (2015); G. Buchalla, O. Cata, A. Celis, and C. Krause, Phys. Lett. B 750, 298 (2015); D. Espriu and F. Mescia, Phys. Rev. D 90, 015035 (2014).

[18] R. L. Delgado, A. Dobado, and F. J. Llanes-Estrada, J. High Energy Phys. 02 (2014) 121; Phys. Rev. Lett. 114, 221803 (2015); Phys. Rev. D 91, 075017 (2015); D. Espriu, F. Mescia, and B. Yencho, Phys. Rev. D 88, 055002 (2013).

[19] G. Altarelli and R. Barbieri, Phys. Lett. B 253, 161 (1991); see also M. Peskin and T. Takeuchi, Phys. Rev. Lett. 65, 964 (1990) and A. Dobado, D. Espriu, and M. J. Herrero, Phys. Lett. B 255, 405 (1991); see Appendix A on M. Baak, M. Goebel, J. Haller, A. Hoecker, D. Ludwig, K. Moenig, M. Schott, and J. Stelzer, Eur. Phys. J. C 72, 2003 (2012).

[20] I. Brivio, T. Corbett, O. J. P. Eboli, M. B. Gavela, J. Gonzalez-Fraile, M. C. Gonzalez-Garcia, L. Merlo, and S. Rigolin, J. High Energy Phys. 03 (2014) 024.

[21] M. Baak, M. Goebel, J. Haller, A. Hoecker, D. Kennedy, R. Kogler, K. Mönig, M. Schott, and J. Stelzer, Eur. Phys. J. C 72, 2205 (2012). 